\title{
Short-term exposure to intermittent hypoxia leads to changes in gene expression seen in
} chronic pulmonary disease

Gang Wu ${ }^{1 *}$, Yin Yeng Lee ${ }^{1,2 *}$, Evelyn M. Gulla ${ }^{3}$, Andrew Potter ${ }^{4}$, Joseph Kitzmiller ${ }^{5}$, Marc D Ruben $^{1}$, Nathan Salomonis ${ }^{2,6}$, Jeffrey A. Whitsett ${ }^{5}$, Lauren J Francey ${ }^{1}$, John B Hogenesch ${ }^{1}$, David F. Smith $3,7,8,9$

*Both authors contributed equally.

$8{ }^{1}$ Divisions of Human Genetics and Immunobiology, Center for Chronobiology, Department of

9 Pediatrics, Cincinnati Children's Hospital Medical Center, Cincinnati, OH 45229, USA

$10{ }^{2}$ Department of Pharmacology and Systems Physiology, University of Cincinnati College of

11 Medicine, Cincinnati, OH 45229, USA

$12{ }^{3}$ Division of Pediatric Otolaryngology-Head and Neck Surgery, Cincinnati Children's Hospital

13 Medical Center, Cincinnati, OH 45229, USA

$14{ }^{4}$ Division of Developmental Biology, Cincinnati Children's Hospital Medical Center, Cincinnati, $15 \mathrm{OH} 45229$, USA

$16{ }^{5}$ Division of Pulmonary Biology, Cincinnati Children's Hospital Medical Center, Cincinnati, $\mathrm{OH}$ 17 45229, USA

$18{ }^{6}$ Division of Biomedical Informatics, Cincinnati Children's Hospital Medical Center, Cincinnati, $19 \mathrm{OH} 45229$, USA

$20{ }^{7}$ Division of Pulmonary Medicine, Cincinnati Children's Hospital Medical Center, Cincinnati, $\mathrm{OH}$ 21 45229, USA

$22{ }^{8}$ The Sleep Center, Cincinnati Children's Hospital Medical Center, Cincinnati, OH 45229, USA

$23{ }^{9}$ Department of Otolaryngology-Head and Neck Surgery, University of Cincinnati College of 24 Medicine, Cincinnati, OH 45267, USA

\section{Corresponding Author:}

26 David F Smith, MD, PhD

27 Assistant Professor

28 Divisions of Pediatric Otolaryngology, Pulmonary Medicine, and the Sleep Center

29 Cincinnati Children's Hospital Medical Center

30 Department of Otolaryngology-Head and Neck Surgery

31 University of Cincinnati School of Medicine

32 Cincinnati, $\mathrm{OH} 45229$

33 Phone: (513) 803-4194

34 Fax: (513) 636-8133

35 Email: David.Smith3@cchmc.org

Keywords: obstructive sleep apnea, single cell sequencing, intermittent hypoxia, lung, RNA sequencing 


\section{Abstract}

44 Obstructive sleep apnea (OSA) results from episodes of airway collapse and intermittent

45 hypoxia and is associated with a host of health complications including dementia, diabetes,

46 heart failure, and stroke. Although the lung is the first organ to sense changes in inspired

47 oxygen levels, little is known about the consequences of $\mathrm{IH}$ to the lung hypoxia-inducible factor

48 (HIF)-responsive pathways. Furthermore, cellular mechanisms causing disease progression

49 across multiple systems in OSA are unknown. We hypothesized that exposure to IH would lead

50 to up- and down-regulation of diverse expression pathways and that individual cell populations

51 would show distinctive responses to $\mathrm{IH}$. We identify changes in circadian and immune pathways

52 in lungs from mice exposed to $\mathrm{IH}$. Among all cell types, endothelial cells show the most

53 prominent transcriptional changes. Interestingly, up-regulated genes in endothelial, fibroblast,

54 and myofibroblast cells were enriched for genes associated with pulmonary fibrosis and

55 pulmonary hypertension. These genes include targets of several drugs currently used to treat

56 chronic pulmonary diseases. Our results reveal potential candidates for cell-targeted therapy

57 seeking to minimize pulmonary effects of OSA. A better understanding of the pathophysiologic

58 mechanisms underlying diseases associated with OSA could improve our therapeutic

59 approaches, directing therapies to the most relevant cells and molecular pathways.

\section{Introduction}

61 Obstructive sleep apnea (OSA) is a condition characterized by episodes of sleep-associated

62 upper airway obstruction and intermittent hypoxia (IH). OSA occurs in approximately $2-5 \%$ of

63 children(Marcus et al., 2012) and 33\% of adults 30-69 years of age(Benjafield et al., 2019) in

64 the US. If untreated, OSA is associated with significant health consequences to the

65 cardiovascular, neurological, and metabolic systems. Even young children with moderate to

66 severe OSA can develop blood pressure dysregulation(Amin et al., 2004), systemic

67 hypertension(Enright et al., 2003; Kohyama et al., 2003), and left ventricular hypertrophy(Amin 
et al., 2002, 2005). OSA is associated with a significant socioeconomic burden in the US(Sullivan, 2016). Despite available medical and surgical therapies, millions of children and adults with OSA are currently untreated or do not respond to available therapies. Cellular responses to changes in oxygen levels are primarily mediated by the hypoxia-inducible factors (HIFs). Although the lung is the first organ to sense large changes in inspired oxygen levels,

73 little is known about the consequences of IH to the lung HIF-responsive pathways. Without a

74 basic understanding of the molecular mechanisms that lead to diseases associated with $\mathrm{IH}$ and

75 OSA, our ability to identify new treatments is significantly hindered. inflammation(Gozal et al., 2008), oxidative stress(Tauman et al., 2014), and endothelial dysfunction(Bhattacharjee et al., 2012; Kheirandish-Gozal et al., 2013). However, the early causal events from $\mathrm{IH}$-exposure are not fully elucidated. Research is now focused on other possible pathogenic pathways that could be activated or suppressed in the presence of $\mathrm{IH}$,

81 leading to disease. For example, hypoxia inducible factors (HIFs) stabilized under low oxygen

82 conditions can affect the circadian transcriptional-translational feedback loop at the cellular

83 level(Adamovich et al., 2017; Hogenesch et al., 1998; Kobayashi et al., 2017; Peek et al., 2017;

84 Wu et al., 2017). Even acute exposure to IH results in dysregulation of the circadian clock that is 85 time-of-day-dependent and tissue-specific, and these effects persist in some tissue for up to 24

86 hours after exposure(Manella et al., 2020). Pathways involved in immune responses and

87 regulation can also be activated or suppressed in the presence of $\mathrm{IH}$ (Cubillos-Zapata et al.,

88 2017; Lam \& Ip, 2019), contributing to comorbid disease initiation and progression. Associations

89 between $\mathrm{IH}$ and gene targets could be either pathogenic or protective responses for the lung.

90 Additionally, lung could be an effector rather than target organ of $\mathrm{IH}$, resulting in responses to $\mathrm{IH}$ 91 that lead to multi-systemic effects. 
While animal models of OSA have focused on physiologic responses to $\mathrm{IH}$ at organ and

93 system levels, determination of the contributions of individual cell types in initiation and

94 progression of disease has been challenging. Within organs, individual cells serve specific

95 physiologic roles. As a result, pathways disrupted by stabilization of HIFs can affect cell types

96 differently. Single-cell RNA sequencing (scRNA-seq) has emerged as a method for evaluating

97 transcriptional states from thousands of individual cells(M. J. Zhang et al., 2018), advancing our

98 understanding of how specific cell types contribute to physiology and disease(Plasschaert et al.,

99 2018; M. J. Zhang et al., 2018).

In the present study, we used IH as a mouse model of OSA to better understand early

101 cellular-specific consequences to the lung, the primary organ that first senses hypoxic episodes.

102 We hypothesized that exposure to $\mathrm{IH}$ would lead to up- and down-regulation of diverse

103 expression pathways, distinct cell populations would show distinctive responses to $\mathrm{IH}$, and that

104 changes in these gene expression pathways could provide therapeutic targets at the cell-

105 specific level. We identify changes in both circadian and immune response pathways in lungs

106 from mice exposed to $\mathrm{IH}$. We also demonstrate strong similarities in the gene expression

107 profiles from mice compared to those characteristics of human lung tissue from patients with

108 diverse pulmonary diseases, including pulmonary hypertension and pulmonary fibrosis. Our

109 results reveal potential candidates for cell-targeted therapy seeking to minimize effector

110 responses of the lung that could lend to systemic disease. A better understanding of the

111 pathophysiologic mechanisms underlying diseases associated with OSA could improve our

112 therapeutic approaches.

\section{Results}

114 Short-term exposure to intermittent hypoxia reshapes circadian and immune pathways in 115 the lung 
116 In humans, moderate to severe OSA is associated with interstitial lung disease with remodeling

117 of the extracellular matrix(Kim et al., 2017). Lung is the primary organ that senses episodes of

118 hypoxia and is therefore exposed to large fluctuations in the oxygen concentrations compared to

119 other tissues throughout the body. For these reasons, we sought to identify initial changes in

120 gene expression pathways in the lung in response to $\mathrm{IH}$.

Mice were initially entrained to the same light:dark schedule to synchronize active and inactive phases. After 14 days of entrainment in the $12 \mathrm{~h}: 12 \mathrm{~h}$ light:dark cycle, mice were exposed to $\mathrm{IH}$ or room air (normoxia) for the entire $12 \mathrm{~h}$ inactive phase for 9 days (Fig. 1A). Mice

124 tolerated the procedure well. Furthermore, haemotoxylin and eosin (H\&E) staining of whole

125 lungs did not show gross changes in architecture or inflammatory remodeling after exposure to

126 IH (Fig. S1). Bulk RNA-seq was performed to explore transcriptomic effects of IH on lung tissue

127 at the organ level. There were 374 genes (Fig. S2A) up-regulated and 149 down-regulated in

128 mouse lung after exposure to $\mathrm{IH}(\mathrm{BHQ}<0.05$ and fold change $>1.5)$. Not surprisingly, the top

129 up-regulated genes included well-known hypoxia inducible factor (HIF)-1 target genes (e.g.

130 Edn1, Bnip3 and Ankrd37; Fig. S2B). processes associated with the top 200 up and down regulated genes from mice exposed to $\mathrm{IH}$.

133 Pathways induced in response to hypoxia included circadian rhythm, angiogenesis, and

134 extracellular matrix organization (Fig. 1B and C). Increased expression of RNAs associated with 135 angiogenesis, such as vascular endothelial growth factor (VEGF), was observed after IH, 136 consistent with findings in a mouse model of prolonged exposure to $\mathrm{IH}($ Reinke et al., 2011) and 137 after $72 \mathrm{~h}$ of $\mathrm{IH}$ exposure to endothelial cells in vitro(Wohlrab et al., 2018). Unexpectedly, RNAs 138 associated with immune responses were significantly down regulated after 9 days of IH (Fig. 1B 139 and C). Present findings contrast with the general concept that HIFs are important regulators of 140 inflammation and immune responses (Eltzschig \& Carmeliet, 2011; Scholz \& Taylor, 2013; C. T. 
141 Taylor et al., 2016). For example, activation of neutrophils by HIFs is largely considered

142 proinflammatory(Peyssonnaux et al., 2005; C. T. Taylor et al., 2016; Walmsley et al., 2005). As

143 previously reported, there is a tight interaction between clock genes and HIFs(Edgar et al.,

144 2012; Gu et al., 2000; Hogenesch et al., 1998; Mclntosh et al., 2010; B. L. Taylor \& Zhulin,

145 1999). Several circadian clock repressors (e.g. Nr1d1, Nr1d2, Bhlhe40 and Per3) were

146 significantly upregulated in the IH group (Fig. 1C).

147 Single cell sequencing identifies 19 distinct cell types in the lungs of intermittent hypoxia

148 and control mice

149 We detected significant differences related to a number of cell-type selective genes on

150 biological functions. We then applied single cell transcriptomics to identify cell-type specific

151 effects of IH. In total, we sequenced 12,324 and 16,125 pulmonary cells from IH and control

152 mice. Unsupervised analysis identified 25 transcriptionally distinct cell clusters, corresponding to

15319 distinct cell types (Fig. 2A-C) based on the expression of established marker genes (See

154 Methods), including stromal, epithelial, endothelial, immune, as well as small numbers of other

155 cell types. The proportion of endothelial, AT2, fibroblast/myofibroblast cells were modestly

156 increased, but the proportion of immune cells (e.g. B and T cells) was decreased in the IH

157 exposed mice (Fig. 2C). Overall, the variation of lung cell types was small (BHQ > 0.05). Given

158 that this was a short exposure of $\mathrm{IH}$, we did not anticipate a dramatic difference in cell types or

159 proportions. We then examined samples to determine if lungs from control vs experimental mice

160 showed histologic differences in cell types.

161 Short-term exposure to intermittent hypoxia did not lead to histologic changes in the

162 lung

163 Similar to H\&E histology, confocal immunofluorescence microscopy for major cell types were

164 normal without inflammatory remodeling. Immunostaining for endothelial markers LYVE1 and

165 FOXF1 did not show changes in IH exposed mice (Fig. 3C-D) compared to normoxia (Fig. 3A- 
B). Also important, staining for MKI67 did not show changes in endothelial cell proliferation (Fig. 3E-H). Expression levels of HOPX and SFTPC in alveolar type I and type II cells were not significantly different for IH (Fig. 3K-L) versus control (Fig. 3I-J) mice. Immunostaining for the progenitor marker SOX9 (Fig. 3I-L) or the extracellular matrix marker POSTN (Fig. 3M-P) did not demonstrate any changes after IH exposure. Overall, there were no differences in endothelial or epithelial cells, signs of fibrosis, or increases in the number of progenitor or proliferating cells. Although other studies have demonstrated changes in proliferating type II

173 alveolocytes(Reinke et al., 2011) and pulmonary vascular remodeling(Nisbet et al., 2009), these

174 were chronic mouse models of IH involving months of exposure. These models also produced

175 other phenotypic changes, such as increased lung volumes(Reinke et al., 2011) and pulmonary hypertension(Nisbet et al., 2009). We exposed our mice to $\mathrm{IH}$ for a shorter period of time to specifically evaluate the changes in gene expression prior to lung remodeling with the hope of uncovering early pathways that lead to disease.

Diverse expression pathways were up and down regulated in the presence of intermittent hypoxia

181 We further explored the early cell-type specific response to $\mathrm{IH}$ in mouse lung by aggregating single cell data into "pseudo-bulk" data to compare biological replicates for each identified cell type (see Methods for details). Using DESeq2(Love et al., 2014), the number of up or down regulated genes in different lung cell types in response to $\mathrm{IH}$ are not equal (Fig. S3). To balance the difference in the pathway enrichment analysis, we selected the top 200 up and down regulated genes (ranking by the $P$ value) in each cell type. From the DAVID enrichment analysis, diverse biological processes were up and down regulated in different cell types in response to $\mathrm{IH}$ (Fig. 4A, Fig. S4 A and B). For example, hypoxia-responsive and circadian pathways were enriched in those up regulated genes in response to $\mathrm{IH}$ in endothelial cells,

190 myofibroblasts, and AT2 cells. Immune response-related and antigen processing and 
191 presentation were enriched in those down regulated genes in monocytes, macrophages-

192 dendritic cells, NK cells, and erythroblasts. Surprisingly, circadian pathways were highly

193 enriched in multiple cell populations, not just epithelial cells, a population that is important for

194 circadian rhythmicity in the lung(Gibbs et al., 2009). As expected from specific genes in each

195 biological process, the response level of the same genes were different in multiple cell types

196 (Fig. 4B and C). For example, the circadian repressor gene, Nr1d1, was more responsive to $\mathrm{IH}$

197 in endothelial and AT2 cells than lymphatic endothelial cells and AT1 cells. We also noted cell-

198 specific responses for the down regulated genes. For example, immune response genes (e.g.

$199 \mathrm{Ig} / \mathrm{c} 3, \mathrm{S100a8}$, and Oas3) decreased more in monocytes than macrophages in response to $\mathrm{IH}$.

200 Pulmonary vascular endothelial subpopulations show distinctive responses to

201 intermittent hypoxia

202 Recent studies show distinct vascular endothelial cell subpopulations in mouse and human

203 lung(Ren et al., 2019). Our vascular endothelial populations were annotated to endothelial

204 artery, vein, capillary aerocytes (Cap-a), and general capillary (Cap-g) cells (Fig. 5A and Fig.

205 S5). Interestingly, we found that endothelial cells demonstrated profound changes in gene

206 expression profiles in response to $\mathrm{IH}$. The endothelial capillary cells were more responsive to $\mathrm{IH}$

207 compared to endothelial artery and vein cells (Fig. 5B). For example, at $\mathrm{BHQ}<0.2$, more than

208100 genes were significantly up-regulated in capillary aerocytes and general capillary cells.

209 However, only 1 gene in the arterial endothelial cells and 57 genes in the venular endothelial

210 cells were significantly up-regulated at the same cutoff. This trend persisted at other BHQ cut-

211 offs (Fig. S6). General capillary cells are more responsive to hypoxia than capillary aerocytes. In

212 addition, more genes were significantly up and down regulated in general capillary cells (Fig.

213 5B). Hypoxia-responsive genes (e.g. Pdk1, Vegfc, Slc2a1, Pkm, and Flt1) showed higher levels

214 of expression variation (fold change and significance) in general capillary cells than capillary

215 aerocytes (Fig. 5C), demonstrating variation at the subpopulation level. Glycolytic process was 
216 up regulated and cell migration was down regulated in both capillary aerocytes and general

217 capillary cells in response to IH (Fig. 5D and E). Given the association between glycolysis,

218 cytoskeletal remodeling, and cell migration in other cell types(Shiraishi et al., 2015), the similar

219 enrichment trends for these pathways is not surprising. Additionally, without vascular growth

220 associated with chronic $\mathrm{IH}$, glycolysis may be used to meet metabolic demand. Interestingly,

221 changes in the glycolytic process were specific to endothelial cell types (Fig. S4B).

On the other hand, capillary aerocytes and general capillary cells demonstrated more differences in enrichment pathways (Fig. 5D and E). For example, circadian rhythm and

224 regulation of angiogenesis were only enriched for those up and down regulated genes in

225 general capillary cells in response to $\mathrm{IH}$, while phagocytosis and regulation of cell shape were

226 only enriched for those down regulated genes in capillary aerocytes (Fig. 5D and E, table S12).

227 Anatomic location within the pulmonary vasculature, including variable roles in gas exchange,

228 could lead to these differences in response. Alternatively, proximity and interaction with other

229 cell types, such as fibroblasts or immune cells, could also help to explain these findings.

230 Evaluation of the expression pathways at the single cell resolution demonstrated

231 significant changes in multiple cell types. With this information, we wanted to identify potential

232 candidates for therapeutic intervention in response to $\mathrm{IH}$.

233 Pulmonary disease-regulated genes provide clinical implications for OSA at the cell-

234 specific level

235 OSA is associated with an array of pulmonary diseases, such as interstitial lung disease (Kim et

236 al., 2017), idiopathic pulmonary fibrosis (Lancaster et al., 2009), and pulmonary hypertension

237 (Chaouat et al., 1996; Dimitar Sajkov \& McEvoy, 2009). IH led to significantly more up than

238 down regulated genes (Fig. 6A and Fig. S7). IH-induced expression in myofibroblasts,

239 fibroblasts, AT2 cells, basophils, and macrophage-DCs were significantly enriched for chronic

240 obstructive pulmonary disease (COPD)-associated genes, while myofibroblasts and fibroblasts 
241 demonstrated enrichment of genes associated with pulmonary fibrosis. However, the disease

242 genes were not equally expressed or up regulated in these cell types (Fig. 6B). For example,

243 Ptgis is a pulmonary hypertension-associated gene highly expressed in myofibroblasts and

244 fibroblasts compared to other cell types (Fig. S8). Ptgis is also a target gene for Epoprostenol-

245 a drug used for treating pulmonary hypertension(Sitbon \& Vonk Noordegraaf, 2017). The

246 idiopathic pulmonary fibrosis-associated gene, Thbs1(Idell et al., 1989; Kuhn \& Mason, 1995;

247 Yehualaeshet et al., 2000), was highly expressed and more responsive to IH in myofibroblasts

248 compared to other cell types. Msr1(Hersh et al., 2006; Silverman et al., 2002) is a COPD-

249 associated gene which was highly expressed in macrophage-DCs, basophils, and monocytes.

250 These data highlight the similarity of the IH signatures with cell-type specific responses in an

251 array of pulmonary diseases (Fig. 6C, Fig. S8).

252 We further evaluated changes in the interactions of these drug-targeted disease genes

253 in each cell type using CellPhoneDB(Vento-Tormo et al., 2018). There are 32 ligand-receptor

254 interactions found in the database using these drug-targeted disease genes. Overall,

255 myofibroblasts were involved in 98 out of 176 gains of interaction $(B H Q=4.28 e-50)$,

256 demonstrating the significance of this cell type in activating the FGF signaling pathway in early

$257 \quad \mathrm{IH}$-associated responses (Fig. 6D, Fig. S9).

\section{Discussion}

259 OSA results from intermittent episodes of airway collapse and hypoxemia and is associated with

260 dementia(Osorio et al., 2015), diabetes(Punjabi \& Beamer, 2009), hypertension(Marin et al.,

261 2012), heart failure(Gottlieb et al., 2010), and stroke(Valham et al., 2008). How cellular

262 responses to hypoxia and hypoxemia initiate and cause disease progression in multiple organs

263 remains unknown. Using $\mathrm{IH}$ as a mouse model of OSA, we show profound, cell-type specific

264 changes in genome-wide RNA expression in the lung. RNA profiles from lungs of mice exposed

265 to IH shared similarity with gene expression changes in human lung from patients with 
pulmonary disease, including pulmonary hypertension, COPD, and asthma. OSA is associated with injury to alveolar epithelial cells and extracellular matrix remodeling, key features of

268 interstitial lung disease (Kim et al., 2017). Although it is known that pulmonary diseases share

269 general mechanisms, such as systemic inflammation and oxidative stress(McNicholas, 2009),

270 there is an incomplete understanding of the early-stage changes in the lung from OSA.

In the present study, macrophages, dendritic cells, and NK cells were the only

272 populations to demonstrate altered oxidation-reduction in the early stages of IH exposure. It was

273 previously demonstrated that chronic $\mathrm{IH}$ in mice caused the release of free oxygen radicals in

274 the lung(Tuleta et al., 2016), effects that could be blunted with antioxidative agents(Tuleta et al.,

275 2016). Pulmonary hypertension from chronic IH was associated with enhanced NADPH oxidase

276 expression, and knockout mice lacking one of these subunits demonstrated attenuated effects

277 of chronic $\mathrm{IH}$ (Nisbet et al., 2009). Which cells mediate these effects in the lung? This type of

278 knowledge can help direct therapeutics to the most relevant cells and molecular pathways.

In our mice exposed to $\mathrm{IH}$, prostacyclin synthase (Ptgis) expression was dramatically

2862 cells. FGFR2 promotes alveolar regeneration in response to lung injury(Perl \& Gale, 2009),

287 and is upregulated in patients with idiopathic pulmonary fibrosis(Li et al., 2018). Previous

288 studies suggest that OSA can induce injury to the lung(Aihara et al., 2011; Lederer et al., 2009),

289 and OSA is prevalent in patients with idiopathic pulmonary fibrosis(Lancaster et al., 2009). If 
OSA does, in fact, lead to fibrotic changes in the lung, targeting FGF pathways in alveolar

291 epithelial cells could prevent disease progression from IH.

Although there is limited data on the role of the circadian clock in OSA(von Allmen et al., 2018), it may play a significant role in this disease(Butler et al., 2015; Entzian et al., 1996; Smith et al., 2017). We found dysregulation of circadian gene expression in multiple cell types. The circadian clock is a transcriptional/translational feedback loop that coordinates $24 \mathrm{~h}$ timing of physiological functions. BMAL1, the key clock transcription factor(Hogenesch et al., 1998),

297 interacts with CLOCK(King et al., 1997) and it's partner NPAS2(Zhou et al., 1997) to activate

298 hundreds of target genes. All three are members of the basic helix-loop-helix (bHLH)-PER-

299 ARNT-SIM (PAS) transcription factor family. The HIFs (1-3) are members of the same

300 transcription factor family and are stabilized under low oxygen conditions. It is known that the 301 circadian clock in alveolar epithelial cells impacts pulmonary physiology, and it's disruption can contribute to disease in animal models(Z. Zhang et al., 2019). More importantly, IH leads to intertissue circadian misalignment (including in the lung) in mice(Manella et al., 2020). The hypoxic episodes that define OSA are clearly diurnal, but we don't understand if clock disruption is a cause or consequence of disease. Our findings suggest that circadian clock dysfunction may be an important early-stage consequence of hypoxia-driven disease and may contribute to downstream processes. changes. This contrasts with findings from some prior murine models of $\mathrm{IH}$. For example, IH

310 induced epithelial cell proliferation in lungs from a chronic exposure model (Reinke et al., 2011).

311 In a bleomycin-induced lung injury model, fibrosis in mouse lung is worsened by $\mathrm{IH}(\mathrm{Gille}$ et al.,

312 2018). In our model, short-term exposure to $\mathrm{IH}$ did not result in changes to the parenchyma or

313 vessels. This is likely attributed to the difference in the length of time of IH exposure. Future

314 longitudinal studies should address how gene expression profiles change over time and which 
cell types drive disease progression from early to late $\mathrm{IH}$ exposure. Early insults from hypoxia may also drive organ-specific damage in other systems. Although models of IH replicate desaturation and recovery of fractional inhaled oxygen, there is variability in the number of hypoxic events, length of desaturation events, and length of overall exposure. This variability could affect expression profiles and histopathologic findings. in OSA. By identifying the roles of individual cells in disease, we have the opportunity to test targeted therapeutics, focusing specifically on the most pathologically-relevant cells and molecular pathways. Studies using scRNA-seq are already being used to identify novel cell populations in disease. For example, Xu and colleagues(Xu et al., 2016) identified a loss of normal epithelial cells in the development of idiopathic pulmonary fibrosis. In another study using single-cell profiling of bronchial epithelial cells, the major source of cystic fibrosis transmembrane conductance regulator (CFTR) activity, the pulmonary ionocyte, was revealed(Plasschaert et al., 2018). As CFTR is the gene mutated in cystic fibrosis, cell-specific therapies for this disease can now be evaluated. candidates for therapeutic intervention. Given the socioeconomic burden to our healthcare system for diagnosing and treating OSA, new diagnostic and therapeutic strategies will be vital

333 for the coming years.

\section{Materials and Methods}

335 Animals. Use of animals and all procedures were approved by the Institutional Animal Care and

336 Use Committee at Cincinnati Children's Hospital Medical Center and complied with the National 337 Institutes of Health guidelines. Male C57BL/6J wild type mice aged 6 weeks were purchased

338 from The Jackson Laboratory (Bar Harbor, ME) and entrained to a 12h:12h light dark cycle for 2 339 weeks prior to exposure. 
341 housed in light boxes and entrained to a 12:12 light:dark cycle for 2 weeks prior to initiation of

$342 \mathrm{IH}$. Mice were randomly assigned to $\mathrm{IH}$ or room air exposures. For the experimental group, mice

343 were maintained in a commercially-designed gas control delivery system (Model A84XOV,

344 BioSpherix, Parish, NY) during the inactive (light) phase from (ZT 0-12). Mice were provided

345 with food and water ad libitum. For each episode of $\mathrm{IH}$, the fractional inhaled oxygen $\left(\mathrm{O}_{2}\right)$ was

346 reduced from $20.9 \%$ to $6 \%$ over a 50 s exposure period, followed by an immediate $50 \mathrm{~s}$

347 recovery period to $20.9 \%$. The fractional oxygen was maintained at $20.9 \%$ for approximately 15

$348 \mathrm{~s}$ before the cycle was repeated, allowing for $\sim 30$ hypoxic events per hour. Ambient temperature

349 in the hypoxia chamber was maintained between $22-24^{\circ} \mathrm{C}$ to match room air. Mice in the

350 experimental group were maintained at room air during the active phase, ZT 13-24. Mice in the

351 control group were maintained at room air throughout the circadian cycle, ZT 0-24. Experimental

352 and control mice were exposed to IH vs room air for 9 days, followed by sacrifice at ZT 3 on day

35310 of exposure. This was immediately followed by organ harvest and preparation for bulk RNA-

354 seq, scRNA-seq, or histopathology.

355 RNA Isolation. In total, 6 experimental and 6 control mice were used for bulk RNA-seq. For bulk

356 RNA-seq, the lung was quickly harvested and snap-frozen in liquid nitrogen. Organs were later

357 homogenized in TRIzol reagent (Invitrogen) and processed using a bead mill homogenizer

358 (Qiagen Tissuelyser). RNA was then isolated from lung homogenates by phase separation

359 using chloroform and phase separation columns. The aqueous phase was then applied to an

360 RNeasy column following the manufacturer's protocol (Qiagen) to extract and purify the RNA.

361 Bulk RNA Sequencing and Analysis. RNA from the lungs of control and experimental mice were

362 sent for bulk sequencing separately. Approximately 0.4 ug of total RNA was used for library

363 preparation. mRNA enrichment and library preparation was performed using the Polyadenylated

364 (PolyA+) mRNA Magnetic Isolation Module (New England Biolabs) and NEBNext Ultra II RNA

365 Library Prep Kit for Illumina (New England Biolabs), following the manufacturer's protocol. All 12 
samples were then pooled together and sequenced in one lane using Illumina Novaseq 6000 platform with paired-end 150bp (table S1). The raw fastq files from RNA-seq were mapped to GRCm38 mouse genome reference using STAR (version 2.5) with default parameters. More than $90 \%$ (table S1) of sequenced paired-end reads (above 50M reads for each library) were mapped to the mouse genome by STAR(Dobin et al., 2013). HTSeq(Anders et al., 2015)

371 (version 0.6.0) was used to quantify gene expression, with Ensembl GRCm38.96 as a

372 reference. DESeq2 (version 1.24.0) was used to perform the differential expression analysis on

373 the HTSeq quantified count per gene. The top 200 up and down regulated genes (table S2),

374 ranked by P-value from low to high with fold-change above 1.5 (or log2(fold-change) $>0.58$ ),

375 were used for biological process enrichment analysis in the DAVID database. Biological process

376 terms with at least 5 differentially expressed genes and $\mathrm{BHQ}<0.15$ were selected. For

377 aggregating redundant biological processes, GOSemSim(Yu et al., 2010) (version 2.10.0) was

378 used to calculate the semantic similarity ('Jiang' method from GOSemSim) between significant

379 biological processes. Redundant biological processes were manually merged into biological

380 process categories (table S3).

381 Dissociation Protocol for Single Cell Sequencing. For scRNA-seq, a total of 3 biological

382 replicates (3 $\mathrm{IH}$ and 3 controls in the first experiment and 2 of each in the other replicates). On

383 the last day of exposure, the mice were sacrificed, lung harvested, and tissue immediately

384 placed in ice-cold PBS. Dissociation of the pooled whole mouse lung for each group (IH vs RA)

385 was performed as previously described(Guo et al., 2019). Briefly, minced lung was placed in

386 collagenase/elastase/dispase digestion buffer (Sigma-Aldrick, St. Louis, MO; Worthington

387 Biochemical, Lakewood, NJ). After mixing on ice for approximately 3 minutes, the lung was

388 minced again. After resting the suspension, the supernatant was passed through a $30 \mu \mathrm{M}$ filter.

389 A Bacillus licheniformis mix (Sigma-Aldrich) was added to the cell suspension, mixed on ice for

390 approximately $10 \mathrm{~min}$, and passed through a $30 \mu \mathrm{M}$ filter. The suspension was spun at $500 \mathrm{~g}$ for

$3915 \mathrm{~min}$ at $4^{\circ} \mathrm{C}$. The pellet was rinsed with a red blood cell lysis buffer. This was again passed 
through a $30 \mu \mathrm{M}$ filter and then spun at $500 \mathrm{~g}$ for $5 \mathrm{~min}$ at $4^{\circ} \mathrm{C}$. The cell suspension was resuspended in PBS/BSA and manually counted with a hemocytometer. The volume was

394 adjusted to obtain a final concentration of approximately 1000 cells/ $\mu \mathrm{L}$ to be loaded to the $10 \mathrm{X}$

395 Chromium platform.

396 scRNA-seq Library Construction and Sequencing. The single cell suspension was applied to the 397 10X Genomics Chromium platform (San Francisco, CA) to capture and barcode cells, as

398 described in the manufacturer's protocol. Libraries were constructed using the Single Cell 3'

399 Reagent Kit (v2 Chemistry). The completed libraries were then sequenced using HiSeq 2500

400 (Illumina, San Diego, CA) running in Rapid Mode. Each sample was loaded onto two lanes of a

401 Rapid v2 flow cell.

402 scRNA-seq Data Processing. Raw data from 10X Genomics were demultiplexed and converted

403 to a fastq file using cellRanger (v2.1.1) mkfastq. Reads from the same library sequenced in

404 different flow cells (technical replicates) were combined and aligned to the mm10 genome

405 reference using cellRanger count. Summary sentences for statistical mapping are presented in

406 table S4. The gene expression profiles for cells from the three biological replicates of the IH 407 group were combined with cellRanger aggr and were run an unsupervised analysis using the 408 software Iterative Clustering and Guide-gene Selection (ICGS) versions 2 (AltAnalyze version

409 2.1.2) to generate reference clusters using the program defaults with euclidean

410 clustering(DePasquale et al., 2019). ICGS2 grouped 12,324 cells into 25 reference clusters

411 based on the expression profiles of 1,480 selected marker genes (table S5). All cells from

412 control and IH groups were then aligned to these 25 reference clusters using

413 cellHarmony(DePasquale et al., 2019). Uniform Manifold Approximation and Projection (UMAP)

414 calculation was run using integrated function in AltAnalyze -v2.1.2 with default parameters. For

415 annotating the 25 reference clusters into known lung cell-types, we prepared a comprehensive

416 marker gene list for known lung cell types. The sources of this marker gene list included

417 information from the Mouse Cell Atlas, ToppGene, and Lung Gene Expression Analysis 
418 (LGEA)(Chen et al., 2009; Consortium et al., 2018; Du et al., 2017). Additionally, we manually 419 collected cell marker genes from published scRNA-seq studies performed in mouse or human

420 lung(Guo et al., 2019; Zilionis et al., 2019). One-tailed Fisher exact test was used to perform

421 enrichment analysis between marker genes for each cluster and the curated reference markers

422 of known lung cell types. Each cluster was manually assigned to a specific cell type based on

423 the known cell type with the lowest BH(Benjamini \& Hochberg, 1995) adjusted P-value (GO-

424 Elite software)(Zambon et al., 2012). Those clusters corresponding to the same annotated cell

425 type were manually joined as one cell type for downstream analyses (e.g. endothelial

426 corresponding to four clusters). This process reduced the 25 reference clusters into 19 cell

427 types (table S6). For testing the cell type composition difference of mouse lung between

428 experimental and control groups, a centered log ratio transformation was performed on the

429 percentage of each cell type before applying the t-test (two-tailed). The statistical P-value from

430 the t-test was adjusted with the $\mathrm{BH}$ method.

431 Pseudo-bulk RNA-seq Differential Expression Analysis. To identify differentially expressed

432 genes in each lung cell type between the control and IH groups with multiple biologic replicates,

433 all cells assigned to the same cell type were aggregated into a "pseudo-bulk" data library by

434 library. For each library, the sum of the reads per gene from cells assigned to the same cell type

435 were used to represent the cell-type-specific gene expression profiles. Percentage of cells

436 expressed per gene were calculated as a fraction of cells with $>=1$ read(s) for the gene in each

437 cell-type. Count per one million UMI (CPM) for each cell type was calculated as the (sum of

438 reads per gene / sum of reads) * 1000000 for each library. Differential expression analysis was

439 performed with DESeq2 for each cell-type, using the sum of reads per gene as input, with three

440 replicates in each of the control and IH groups. Ranked by P-value from low to high, the top 200

441 up and down regulated genes (table S7) with fold-change above 1.2 (or log2(fold-change) >

442 0.26) were used for biological process enrichment analysis in the DAVID database. Selecting 
443 and aggregating biological processes (table S8) were performed as described above in the

444 Methods section labeled, "Bulk RNA Sequencing and Analysis." We further extracted those

445 genes enriched in circadian rhythm and immune response, and selected well-established genes

446 in each biological process to demonstrate their expression variation under IH exposure in each

447 cell type based on literature searches.

448 Endothelial Subpopulations Analysis. To improve the accuracy for classifying endothelial cells,

449 we reran the AltAnalyze-ICGS2 clustering algorithm using only the 5,579 cells annotated to

450 pulmonary vascular endothelial cells from IH exposure groups, followed by a realignment of all

451 vascular endothelial cells from both control and IH groups to these clusters using cellHarmony.

452 AltAnalyze-ICGS2 produced 6 clusters with 314 marker genes (table S9). We matched the

453 ICGS2 selected marker genes with vascular endothelial subpopulation marker genes presented

454 in a study from Travaglini et al(Travaglini et al., 2019). This annotated the clusters into 4

455 subpopulations of endothelial artery, vein, capillary aerocytes, and general capillary cells (table

$456 \mathrm{~S} 10)$. We further aggregated all cells of the same vascular endothelial subpopulations into

457 "pseudo-bulk" data library by library. DESeq2 was used to detect differentially expressed genes

458 for each subpopulation between the control and IH exposure groups. To select differentially

459 expression genes in each endothelial subpopulation, the cut-off was set as $\mathrm{BHQ}<0.2$ and fold-

460 change $>1.2$ (or log2(fold-change) $>0.26$ ). Those differential expression genes (table S11) in

461 endothelial capillary cells were used for BP enrichment analysis in the DAVID database.

462 Selecting and aggregating biological processes (table S12) were performed as described above

463 in the Methods section labeled, "Bulk RNA Sequencing and Analysis."

464 Association Analysis on IH Responsive Genes with Pulmonary Disease Genes and Drug

465 Targets at the Cell-type Level. Gene-disease association information was downloaded from the

466 DisGeNET(Piñero et al., 2017) database (curated gene-disease associations). The downloaded

467 file was filtered with keywords to specifically select genes linked to pulmonary diseases, which 
468 includes“allergic asthma”, "asthma”, "chronic obstructive airway disease”, "pulmonary

469 hypertension", "chronic thromboembolic pulmonary hypertension", "idiopathic pulmonary arterial

470 hypertension", "familial primary pulmonary hypertension”, “idiopathic pulmonary hypertension”,

471 "interstitial lung fibrosis", "pulmonary fibrosis", and “idiopathic pulmonary fibrosis". One-tailed

472 Fisher exact test was used to determine if the top 200 up or down regulated genes in each cell-

473 type exposed to IH significantly overlapped with these pulmonary disease associated genes

474 (table S13). The significant cutoff was set with a $\mathrm{BH}$ adjusted $\mathrm{P}$-value from a Fisher exact test <

$475 \quad 0.05$ and at least 5 overlapped genes with any pulmonary disease gene set. For the association

476 analysis between $\mathrm{IH}$ responsive genes and pulmonary drug targets, the $\mathrm{xml}$ file was

477 downloaded from the DrugBank(Wishart et al., 2008) (version 5.1.4). The drugbankR package

478 (https://github.com/yduan004/drugbankR) was used to parse the $\mathrm{xml}$ file to get each drug and

479 its target genes. The parsed drug table was linked with the top 200 up or down regulated genes

480 in each cell-type exposed to IH by drug target genes. The drug table was further filtered with

481 respiratory tissue and disease-associated key words (e.g. asthma, lung, bronchus, airway et al.)

482 to keep candidate drugs used to treat pulmonary diseases. The filtered table was manually

483 curated to select drugs mainly indicated to treat pulmonary diseases. In the association

484 analysis, the 'homologene' package (https://github.com/oganm/homologene) was used to find

485 the human-unique homolog of mouse genes. CellPhoneDB is used to predict ligand-receptor

486 interactions between cell-types. The pulmonary diseases relevant drug targeted genes list (Fig

$4876 \mathrm{C}$ ) is used to select ligand-receptor interaction pairs. Genes with unknown drug effects were

488 filtered out from the analysis. Human homolog gene is used for this analysis. Gain of

489 interactions and loss of interactions is calculated by summing up the number of ligand-receptor

490 interactions that were only significantly present in either hypoxia or control $(P$-value $<0.05$ and

491 mean > 0.1), respectively, for each cell pairs comparison. Two-tailed Fisher exact test was used

492 to calculate significance changes of each cell type. Hive plot is made using the HiveR package

493 (https://github.com/bryanhanson/HiveR). 
495 histological staining, mice were sacrificed, and lung inflation fixation was immediately

496 performed. After exposure of the trachea and lungs, the trachea was cannulated with a scalp

497 vein cannula (EXELINT, Redondo Beach, CA), and 10\% neutral buffered formalin was gravity-

498 perfused into the lung at a height of $25 \mathrm{~cm}$. After infusion, the lung was harvested and placed in

499 formalin for 24 hours. Whole lung was then dehydrated in $70 \%$ ethanol and embedded in

500 paraffin. For morphologic evaluation, $5 \mu \mathrm{m}$ thick sections were cut from the paraffin blocks and

501 stained with hematoxylin and eosin.

Immunofluorescence staining on $10 \%$ formalin fixed mouse lung was performed on $5 \mu \mathrm{m}$

503 thick paraffin embedded tissue sections. Tissue slides were melted at $60^{\circ} \mathrm{C}$ for two hours,

504 following rehydration through xylene and alcohol, and finally in PBS. Antigen retrieval was

505 performed in $0.1 \mathrm{M}$ citrate buffer $(\mathrm{pH}$ 6.0) by microwaving. Slides were blocked for 2 hours at

506 room temperature using $4 \%$ normal donkey serum in PBS containing $0.2 \%$ Triton X-100, and

507 then incubated with primary antibodies diluted in blocking buffer for approximately 16 hours at

$508 \quad 4^{\circ} \mathrm{C}$. Primary antibodies included ACTA2 (1:2000, Sigma-Aldrich), EMCN (1:200, R\&D

509 Systems), FOXFI (1:100, R\&D Systems), HOPX (1:100, Santa Cruz Biotechnology), MKI67

510 (1:100, BDBiosciences), POSTN (1:100, ABCAM), Pro-SFTPC (1:1000, Seven Hills

511 Bioreagents), SOX9 (1:100, Millipore), and LYVE1 (1:100, ABCAM). Secondary antibodies

512 conjugated to Alexa Fluor 488, Alexa Fluor 568, or Alexa Fluor 633, were used at a dilution of

513 1:200 in blocking buffer for 1 hour at room temperature. Nuclei were counterstained with DAPI

$514(1 \mu \mathrm{g} / \mathrm{ml})($ Thermo-Fisher). Sections were mounted using ProLong Gold (Thermo-Fisher)

515 mounting medium and coverslipped. Tissue sections were then imaged on an inverted Nikon

516 A1R confocal microscope using a NA 1.27 objective using a 1.2 AU pinhole. Maximum intensity

517 projections of multi-labeled Z stack images were generated using Nikon NIS-Elements software. 
Acknowledgements: We thank K.A. Wikenheiser-Brokamp for her thoughtful discussion and guidance. We thank Bruce J. Aranow, Yan Xu, Minzhe Guo, Kashish Chetal, and Emily R. Miraldi for their thoughtful discussion about scRNA-seq analysis. We would like to thank S.

521 Steven Potter for allowing us to use resources available in his lab. We would also like to thank

522 Kalpana Srivastava for work on sectioning tissue specimens and completing

523 immunofluorescence assays. Grant Funding: This work was supported by Cincinnati Children's

524 Hospital Medical Center, the National Heart, Lung, and Blood Institute (NHLBI; 1 K08

525 HL148551-01), The Triological Society, and the American Society of Pediatric Otolaryngology

526 (ASPO). Author contributions: G.W., Y.Y.L., E.M.G., M.D.R., L.J.F., J.B.H., and D.F.S.

527 designed research; G.W., Y.Y.L., E.M.G., A.P., J.K., L.J.F., and D.F.S performed research;

528 G.W., Y.Y.L., N.S., J.A.W., and J.B.H. contributed analytic tools; G.W., Y.Y.L., E.M.G., M.D.R.,

529 N.S., J.A.W., L.J.F., and D.F.S. analyzed data; G.W., Y.Y.L., M.D.R., L.J.F., J.B.H., and D.F.S.

530 wrote this paper. Data and Materials Availability: All data associated with this study are

531 present in the paper or the Supplementary Materials and have been incorporated into GEO

532 database (GSE145436).

533 Competing Interests: The authors declare that they have no competing interests.

\section{References}

Adamovich, Y., Ladeuix, B., Golik, M., Koeners, M. P., \& Asher, G. (2017). Rhythmic Oxygen Levels Reset Circadian Clocks through HIF1a. Cell Metabolism, 25(1), 93-101.

Aihara, K., Oga, T., Harada, Y., Chihara, Y., Handa, T., Tanizawa, K., Watanabe, K., Tsuboi, T., Hitomi, T., Mishima, M., \& Chin, K. (2011). Comparison of biomarkers of subclinical lung injury in obstructive sleep apnea. Respiratory Medicine, 105(6), 939-945.

Amin, R. S., Carroll, J. L., Jeffries, J. L., Grone, C., Bean, J. A., Chini, B., Bokulic, R., \& Daniels, S. R. (2004). Twenty-four-hour Ambulatory Blood Pressure in Children with Sleepdisordered Breathing. American Journal of Respiratory and Critical Care Medicine, 169(8), 
950-956.

544 Amin, R. S., Kimball, T. R., Bean, J. A., Jeffries, J. L., Willging, J. P., Cotton, R. T., Witt, S. A.,

Glascock, B. J., \& Daniels, S. R. (2002). Left ventricular hypertrophy and abnormal

Amin, R. S., Kimball, T. R., Kalra, M., Jeffries, J. L., Carroll, J. L., Bean, J. A., Witt, S. A., ventricular geometry in children and adolescents with obstructive sleep apnea. American

Anders, S., Pyl, P. T., \& Huber, W. (2015). HTSeq--a Python framework to work with highthroughput sequencing data. In Bioinformatics (Vol. 31, Issue 2, pp. 166-169). https://doi.org/10.1093/bioinformatics/btu638

Bady, E., Achkar, A., Pascal, S., Orvoen-Frija, E., \& Laaban, J. P. (2000). Pulmonary arterial hypertension in patients with sleep apnoea syndrome. Thorax, 55(11), 934-939.

Benjafield, A. V., Ayas, N. T., Eastwood, P. R., Heinzer, R., Ip, M. S. M., Morrell, M. J., Nunez, C. M., Patel, S. R., Penzel, T., Pépin, J.-L., Peppard, P. E., Sinha, S., Tufik, S., Valentine, K., \& Malhotra, A. (2019). Estimation of the global prevalence and burden of obstructive

(1)
sleep apnoea: a literature-based analysis. In The Lancet Respiratory Medicine (Vol. 7, Issue 8, pp. 687-698). https://doi.org/10.1016/s2213-2600(19)30198-5

Benjamini, Y., \& Hochberg, Y. (1995). Controlling the False Discovery Rate: A Practical and Powerful Approach to Multiple Testing. In Journal of the Royal Statistical Society: Series B (Methodological) (Vol. 57, Issue 1, pp. 289-300). https://doi.org/10.1111/j.25176161.1995.tb02031.x

Bhattacharjee, R., Kim, J., Alotaibi, W. H., Kheirandish-Gozal, L., Capdevila, O. S., \& Gozal, D. (2012). Endothelial dysfunction in children without hypertension: potential contributions of obesity and obstructive sleep apnea. Chest, 141(3), 682-691.

Butler, M. P., Smales, C., Wu, H., Hussain, M. V., Mohamed, Y. A., Morimoto, M., \& Shea, S. A. 
(2015). The Circadian System Contributes to Apnea Lengthening across the Night in Obstructive Sleep Apnea. Sleep, 38(11), 1793-1801.

571 Chaouat, A., Weitzenblum, E., Krieger, J., Oswald, M., \& Kessler, R. (1996). Pulmonary

572 hemodynamics in the obstructive sleep apnea syndrome. Results in 220 consecutive 573 patients. Chest, 109(2), 380-386.

574 Chen, J., Bardes, E. E., Aronow, B. J., \& Jegga, A. G. (2009). ToppGene Suite for gene list 575 enrichment analysis and candidate gene prioritization. Nucleic Acids Research, 37(Web $576 \quad$ Server issue), W305-W311.

577 Consortium, T. T. M., The Tabula Muris Consortium, Coordination, O., Coordination, L., Organ 578 collection and processing, Library preparation and sequencing, Analysis, C. D., Annotation, C. T., Writing group, Supplemental text writing group, \& Principal investigators. (2018).

Cubillos-Zapata, C., Avendaño-Ortiz, J., Hernandez-Jimenez, E., Toledano, V., Casas-Martin, J., Varela-Serrano, A., Torres, M., Almendros, I., Casitas, R., Fernández-Navarro, I., Single-cell transcriptomics of 20 mouse organs creates a Tabula Muris. In Nature (Vol. 562, Hypoxia-induced PD-L1/PD-1 crosstalk impairs T-cell function in sleep apnoea. The Issue 7727, pp. 367-372). https://doi.org/10.1038/s41586-018-0590-4

DePasquale, E. A. K., Schnell, D., Dexheimer, P., Ferchen, K., Hay, S., Chetal, K., ValienteAlandí, Í., Blaxall, B. C., Grimes, H. L., \& Salomonis, N. (2019). cellHarmony: cell-level matching and holistic comparison of single-cell transcriptomes. Nucleic Acids Research, 47(21), e138.

Dobin, A., Davis, C. A., Schlesinger, F., Drenkow, J., Zaleski, C., Jha, S., Batut, P., Chaisson, M., \& Gingeras, T. R. (2013). STAR: ultrafast universal RNA-seq aligner. Bioinformatics , 29(1), 15-21. 
Du, Y., Kitzmiller, J. A., Sridharan, A., Perl, A. K., Bridges, J. P., Misra, R. S., Pryhuber, G. S., Mariani, T. J., Bhattacharya, S., Guo, M., Potter, S. S., Dexheimer, P., Aronow, B., Jobe, A. H., Whitsett, J. A., \& Xu, Y. (2017). Lung Gene Expression Analysis (LGEA): an integrative web portal for comprehensive gene expression data analysis in lung development. Thorax, 72(5), 481-484.

Edgar, R. S., Green, E. W., Zhao, Y., van Ooijen, G., Olmedo, M., Qin, X., Xu, Y., Pan, M., 7399, pp. 459-464). https://doi.org/10.1038/nature11088

Enright, P. L., Goodwin, J. L., Sherrill, D. L., Quan, J. R., \& Quan, S. F. (2003). Tucson children's assessment of sleep apnea study. Blood Pressure Elevation Associated with Sleep-Related Breathing Disorder in a Community Sample of White and Hispanic Children: The Tucson Children's Assessment of Sleep Apnea Study. Arch Pediatr Adolesc Med, 157(9), 901-904.

Entzian, P., Linnemann, K., Schlaak, M., \& Zabel, P. (1996). Obstructive sleep apnea syndrome and circadian rhythms of hormones and cytokines. American Journal of Respiratory and Critical Care Medicine, 153(3), 1080-1086.

Farber, H. W., \& Gin-Sing, W. (2016). Practical considerations for therapies targeting the prostacyclin pathway. European Respiratory Review: An Official Journal of the European Respiratory Society, 25(142), 418-430.

Gibbs, J. E., Beesley, S., Plumb, J., Singh, D., Farrow, S., Ray, D. W., \& Loudon, A. S. I. (2009). Circadian timing in the lung; a specific role for bronchiolar epithelial cells. Endocrinology, 150(1), 268-276. 
621 Gille, T., Didier, M., Rotenberg, C., Delbrel, E., Marchant, D., Sutton, A., Dard, N., Haine, L., Voituron, N., Bernaudin, J.-F., Valeyre, D., Nunes, H., Besnard, V., Boncoeur, E., \& Planès, C. (2018). Intermittent Hypoxia Increases the Severity of Bleomycin-Induced Lung Injury in Mice. Oxidative Medicine and Cellular Longevity, 2018, 1240192.

Gottlieb, D. J., Yenokyan, G., Newman, A. B., O’Connor, G. T., Punjabi, N. M., Quan, S. F.,

Gozal, D., Serpero, L. D., Sans Capdevila, O., \& Kheirandish-Gozal, L. (2008). Systemic inflammation in non-obese children with obstructive sleep apnea. Sleep Medicine, 9(3), 254-259.

Guo, M., Du, Y., Gokey, J. J., Ray, S., Bell, S. M., Adam, M., Sudha, P., Perl, A. K., Deshmukh, H., Potter, S. S., Whitsett, J. A., \& Xu, Y. (2019). Single cell RNA analysis identifies cellular heterogeneity and adaptive responses of the lung at birth. Nature Communications, 10(1),

Gu, Y. Z., Hogenesch, J. B., \& Bradfield, C. A. (2000). The PAS superfamily: sensors of environmental and developmental signals. Annual Review of Pharmacology and Silverman, E. K. (2006). Genetic linkage and association analysis of COPD-related traits on chromosome 8p. COPD, 3(4), 189-194.

Hogenesch, J. B., Gu, Y.-Z., Jain, S., \& Bradfield, C. A. (1998). The basic-helix-loop-helix-PAS orphan MOP3 forms transcriptionally active complexes with circadian and hypoxia factors. Proceedings of the National Academy of Sciences, 95(10), 5474-5479.

Huang, D. W., Sherman, B. T., \& Lempicki, R. A. (2009). Systematic and integrative analysis of large gene lists using DAVID bioinformatics resources. Nature Protocols, 4(1), 44-57. 
647

648

649

650

Idell, S., Maunder, R., Fein, A. M., Switalska, H. I., Tuszynski, G. F., McLarty, J., \&

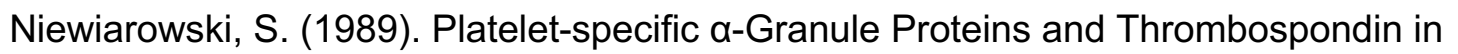
Bronchoalveolar Lavage in the Adult Respiratory Distress Syndrome. In Chest (Vol. 96, Issue 5, pp. 1125-1132). https://doi.org/10.1378/chest.96.5.1125

Kheirandish-Gozal, L., Khalyfa, A., Gozal, D., Bhattacharjee, R., \& Wang, Y. (2013). Endothelial dysfunction in children with obstructive sleep apnea is associated with epigenetic changes in the eNOS gene. Chest, 143(4), 971-977.

Kim, J. S., Podolanczuk, A. J., Borker, P., Kawut, S. M., Raghu, G., Kaufman, J. D., Stukovsky, K. D. H., Hoffman, E. A., Barr, R. G., Gottlieb, D. J., Redline, S. S., \& Lederer, D. J. (2017). Obstructive Sleep Apnea and Subclinical Interstitial Lung Disease in the Multi-Ethnic Study of Atherosclerosis (MESA). Annals of the American Thoracic Society, 14(12), 1786-1795.

King, D. P., Zhao, Y., Sangoram, A. M., Wilsbacher, L. D., Tanaka, M., Antoch, M. P., Steeves, T. D. L., Vitaterna, M. H., Kornhauser, J. M., Lowrey, P. L., \& Others. (1997). Positional cloning of the mouse circadian clockgene. Cell, 89(4), 641-653.

Kobayashi, M., Morinibu, A., Koyasu, S., Goto, Y., Hiraoka, M., \& Harada, H. (2017). A circadian clock gene, PER2, activates HIF-1 as an effector molecule for recruitment of HIF-1a to promoter regions of its downstream genes. The FEBS Journal, 284(22), 3804-3816.

Kohyama, J., Ohinata, J. S., \& Hasegawa, T. (2003). Blood pressure in sleep disordered breathing. Archives of Disease in Childhood, 88(2), 139-142.

Kuhn, C., \& Mason, R. J. (1995). Immunolocalization of SPARC, tenascin, and thrombospondin in pulmonary fibrosis. The American Journal of Pathology, 147(6), 1759-1769.

Lam, D. C. L., \& Ip, M. S. M. (2019). Sleep apnoea and immune regulation: The story is only beginning [Review of Sleep apnoea and immune regulation: The story is only beginning]. Respirology , 24(7), 624-625.

Lancaster, L. H., Mason, W. R., Parnell, J. A., Rice, T. W., Loyd, J. E., Milstone, A. P., Collard, H. R., \& Malow, B. A. (2009). Obstructive sleep apnea is common in idiopathic pulmonary 
fibrosis. Chest, 136(3), 772-778.

674 Lederer, D. J., Jelic, S., Basner, R. C., Ishizaka, A., \& Bhattacharya, J. (2009). Circulating KL-6, 675 a biomarker of lung injury, in obstructive sleep apnoea. The European Respiratory Journal: 676 Official Journal of the European Society for Clinical Respiratory Physiology, 33(4), 793$677 \quad 796$.

678 Li, Li, L., Zhang, S., Wei, L., Wang, Z., Ma, W., Liu, F., \& Qian, Y. (2018). FGF2 and FGFR2 in 679 patients with idiopathic pulmonary fibrosis and lung cancer. In Oncology Letters. https://doi.org/10.3892/ol.2018.8903

Love, M. I., Huber, W., \& Anders, S. (2014). Moderated estimation of fold change and dispersion for RNA-seq data with DESeq2. In Genome Biology (Vol. 15, Issue 12). https://doi.org/10.1186/s13059-014-0550-8

Manella, G., Aviram, R., Bolshette, N., Muvkadi, S., Golik, M., Smith, D. F., \& Asher, G. (2020). Hypoxia induces a time- and tissue-specific response that elicits intertissue circadian clock misalignment. In Proceedings of the National Academy of Sciences (Vol. 117, Issue 1, pp. 779-786). https://doi.org/10.1073/pnas.1914112117

Marcus, C. L., Brooks, L. J., Draper, K. A., Gozal, D., Halbower, A. C., Jones, J., Schechter, M. Academy of Pediatrics. (2012). Diagnosis and management of childhood obstructive sleep apnea syndrome. Pediatrics, 130(3), e714-e755.

Marin, J. M., Agusti, A., Villar, I., Forner, M., Nieto, D., Carrizo, S. J., Barbé, F., Vicente, E., Wei, Y., Javier Nieto, F., \& Jelic, S. (2012). Association Between Treated and Untreated Obstructive Sleep Apnea and Risk of Hypertension. In JAMA (Vol. 307, Issue 20). https://doi.org/10.1001/jama.2012.3418

Mclntosh, B. E., Hogenesch, J. B., \& Bradfield, C. A. (2010). Mammalian Per-Arnt-Sim proteins in environmental adaptation. Annual Review of Physiology, 72, 625-645.

McNicholas, W. T. (2009). Chronic obstructive pulmonary disease and obstructive sleep apnea: 
overlaps in pathophysiology, systemic inflammation, and cardiovascular disease. American Journal of Respiratory and Critical Care Medicine, 180(8), 692-700.

Nisbet, R. E., Graves, A. S., Kleinhenz, D. J., Rupnow, H. L., Reed, A. L., Fan, T.-H. M., intermittent hypoxia-induced pulmonary hypertension in mice. American Journal of

Osorio, R. S., Gumb, T., Pirraglia, E., Varga, A. W., -e. Lu, S., Lim, J., Wohlleber, M. E., Ducca,

Peek, C. B., Levine, D. C., Cedernaes, J., Taguchi, A., Kobayashi, Y., Tsai, S. J., Bonar, N. A., McNulty, M. R., Ramsey, K. M., \& Bass, J. (2017). Circadian Clock Interaction with HIF1a Mediates Oxygenic Metabolism and Anaerobic Glycolysis in Skeletal Muscle. Cell

Perl, A.-K. T., \& Gale, E. (2009). FGF signaling is required for myofibroblast differentiation during alveolar regeneration. American Journal of Physiology. Lung Cellular and Molecular Physiology, 297(2), L299-L308.

\section{2}

Peyssonnaux, C., Datta, V., Cramer, T., Doedens, A., Theodorakis, E. A., Gallo, R. L., HurtadoZiola, N., Nizet, V., \& Johnson, R. S. (2005). HIF-1a expression regulates the bactericidal capacity of phagocytes. In Journal of Clinical Investigation (Vol. 115, Issue 7, pp. 18061815). https://doi.org/10.1172/jci23865

Piñero, J., Bravo, À., Queralt-Rosinach, N., Gutiérrez-Sacristán, A., Deu-Pons, J., Centeno, E., García-García, J., Sanz, F., \& Furlong, L. I. (2017). DisGeNET: a comprehensive platform integrating information on human disease-associated genes and variants. Nucleic Acids 
Research, 45(D1), D833-D839.

Plasschaert, L. W., Žilionis, R., Choo-Wing, R., Savova, V., Knehr, J., Roma, G., Klein, A. M., \& Jaffe, A. B. (2018). A single-cell atlas of the airway epithelium reveals the CFTR-rich pulmonary ionocyte. Nature. https://doi.org/10.1038/s41586-018-0394-6

Punjabi, N. M., \& Beamer, B. A. (2009). Alterations in Glucose Disposal in Sleep-disordered Breathing. American Journal of Respiratory and Critical Care Medicine, 179(3), 235-240.

Reinke, C., Bevans-Fonti, S., Grigoryev, D. N., Drager, L. F., Myers, A. C., Wise, R. A., lung growth in adult mice. American Journal of Physiology. Lung Cellular and Molecular Physiology, 300(2), L266-L273.

Ren, X., Ustiyan, V., Guo, M., Wang, G., Bolte, C., Zhang, Y., Xu, Y., Whitsett, J. A., Kalin, T. V., \& Kalinichenko, V. V. (2019). Postnatal Alveologenesis Depends on FOXF1 Signaling in c-KIT Endothelial Progenitor Cells. In American Journal of Respiratory and Critical Care Medicine (Vol. 200, Issue 9, pp. 1164-1176). https://doi.org/10.1164/rccm.201812-2312oc

Sajkov, D., \& McEvoy, R. D. (2009). Obstructive sleep apnea and pulmonary hypertension. Progress in Cardiovascular Diseases, 51(5), 363-370.

741 Sajkov, D., Wang, T., Saunders, N. A., Bune, A. J., Neill, A. M., \& Douglas Mcevoy, R. (1999). Daytime pulmonary hemodynamics in patients with obstructive sleep apnea without lung disease. American Journal of Respiratory and Critical Care Medicine, 159(5 Pt 1), 15181526.

Scholz, C. C., \& Taylor, C. T. (2013). Targeting the HIF pathway in inflammation and immunity. In Current Opinion in Pharmacology (Vol. 13, Issue 4, pp. 646-653). https://doi.org/10.1016/j.coph.2013.04.009 
cytoskeletal remodeling in human prostate and breast cancer cells. In Oncotarget (Vol. 6,

Silverman, E. K., Palmer, L. J., Mosley, J. D., Barth, M., Senter, J. M., Brown, A., Drazen, J. M.,

Kwiatkowski, D. J., Chapman, H. A., Campbell, E. J., Province, M. A., Rao, D. C., Reilly, J. https://doi.org/10.1086/340316

Sitbon, O., \& Vonk Noordegraaf, A. (2017). Epoprostenol and pulmonary arterial hypertension: 20 years of clinical experience. European Respiratory Review: An Official Journal of the European Respiratory Society, 26(143). https://doi.org/10.1183/16000617.0055-2016

Smith, D. F., Hossain, M. M., Hura, A., Huang, G., McConnell, K., Ishman, S. L., \& Amin, R. S. (2017). Inflammatory Milieu and Cardiovascular Homeostasis in Children with Obstructive Undertreating Obstructive Sleep Apnea Draining Healthcare System. Journal of Clinical Sleep Medicine: JCSM: Official Publication of the American Academy of Sleep Medicine.

768 Tauman, R., Lavie, L., Greenfeld, M., \& Sivan, Y. (2014). Oxidative stress in children with obstructive sleep apnea syndrome. Journal of Clinical Sleep Medicine: JCSM: Official Publication of the American Academy of Sleep Medicine, 10(6), 677-681.

Taylor, B. L., \& Zhulin, I. B. (1999). PAS domains: internal sensors of oxygen, redox potential, and light. Microbiology and Molecular Biology Reviews: MMBR, 63(2), 479-506.

\section{Taylor, C. T., Doherty, G., Fallon, P. G., \& Cummins, E. P. (2016). Hypoxia-dependent} regulation of inflammatory pathways in immune cells. The Journal of Clinical Investigation,

776 Travaglini, K. J., Nabhan, A. N., Penland, L., \& Sinha, R. (2019). A molecular cell atlas of the 
human lung from single cell RNA sequencing. bioRxiv.

Tuleta, I., Stöckigt, F., Juergens, U. R., Pizarro, C., Schrickel, J. W., Kristiansen, G., Nickenig, Increased Oxidative Stress, Inflammation, and Disbalance in Protease/Antiprotease System. Lung, 194(6), 1015-1020.

Valham, F., Mooe, T., Rabben, T., Stenlund, H., Wiklund, U., \& Franklin, K. A. (2008). Increased Risk of Stroke in Patients With Coronary Artery Disease and Sleep Apnea. In Circulation (Vol. 118, Issue 9, pp. 955-960). https://doi.org/10.1161/circulationaha.108.783290

Vento-Tormo, R., Efremova, M., Botting, R. A., Turco, M. Y., Vento-Tormo, M., Meyer, K. B., Park, J.-E., Stephenson, E., Polański, K., Goncalves, A., Gardner, L., Holmqvist, S., Henriksson, J., Zou, A., Sharkey, A. M., Millar, B., Innes, B., Wood, L., Wilbrey-Clark, A., ... Teichmann, S. A. (2018). Single-cell reconstruction of the early maternal-fetal interface in humans. Nature, 563(7731), 347-353.

von Allmen, D. C., Francey, L. J., Rogers, G. M., Ruben, M. D., Cohen, A. P., Wu, G., Schmidt, and Neck Surgery, 194599818797311.

Walmsley, S. R., Print, C., Farahi, N., Peyssonnaux, C., Johnson, R. S., Cramer, T., Hypoxia-induced neutrophil survival is mediated by HIF-1alpha-dependent NF-kappaB activity. The Journal of Experimental Medicine, 201(1), 105-115.

Wishart, D. S., Knox, C., Guo, A. C., Cheng, D., Shrivastava, S., Tzur, D., Gautam, B., \& Hassanali, M. (2008). DrugBank: a knowledgebase for drugs, drug actions and drug targets. Nucleic Acids Research, 36(Database issue), D901-D906. 
Wohlrab, P., Soto-Gonzales, L., Benesch, T., Winter, M. P., Lang, I. M., Markstaller, K., Tretter, V., \& Klein, K. U. (2018). Intermittent Hypoxia Activates Duration-Dependent Protective and Injurious Mechanisms in Mouse Lung Endothelial Cells. Frontiers in Physiology, 9, 1754.

Wu, Y., Tang, D., Liu, N., Xiong, W., Huang, H., Li, Y., Ma, Z., Zhao, H., Chen, P., Qi, X., \&

Xu, Y., Mizuno, T., Sridharan, A., Du, Y., Guo, M., Tang, J., Wikenheiser-Brokamp, K. A., Perl,

Yehualaeshet, T., O’Connor, R., Begleiter, A., Murphy-Ullrich, J. E., Silverstein, R., \& Khalil, N. (2000). A CD36 synthetic peptide inhibits bleomycin-induced pulmonary inflammation and connective tissue synthesis in the rat. American Journal of Respiratory Cell and Molecular Biology, 23(2), 204-212.

Yu, G., Li, F., Qin, Y., Bo, X., Wu, Y., \& Wang, S. (2010). GOSemSim: an R package for measuring semantic similarity among GO terms and gene products. In Bioinformatics (Vol. 26, Issue 7, pp. 976-978). https://doi.org/10.1093/bioinformatics/btq064 Begley, N., Saer, B., Wang, P., Cunningham, P., Baxter, M., Durrington, H., Blaikley, J. F., Hussell, T., Rattray, M., Hogenesch, J. B., Gibbs, J., ... Loudon, A. S. I. (2019). Genomewide effect of pulmonary airway epithelial cell-specific Bmal1 deletion. The FASEB Journal, 
830 Zhou, Y.-D., -D. Zhou, Y., Barnard, M., Tian, H., Li, X., Ring, H. Z., Francke, U., Shelton, J.,

831 Richardson, J., Russell, D. W., \& McKnight, S. L. (1997). Molecular characterization of two

832 mammalian bHLH-PAS domain proteins selectively expressed in the central nervous

833 system. In Proceedings of the National Academy of Sciences (Vol. 94, Issue 2, pp. 713-

834 718). https://doi.org/10.1073/pnas.94.2.713

835 Zilionis, R., Engblom, C., Pfirschke, C., Savova, V., Zemmour, D., Saatcioglu, H. D., Krishnan,

836 I., Maroni, G., Meyerovitz, C. V., Kerwin, C. M., Choi, S., Richards, W. G., De Rienzo, A.,

837 Tenen, D. G., Bueno, R., Levantini, E., Pittet, M. J., \& Klein, A. M. (2019). Single-Cell

838 Transcriptomics of Human and Mouse Lung Cancers Reveals Conserved Myeloid

839 Populations across Individuals and Species. Immunity, 50(5), 1317-1334.e10. 
bioRxiv preprint doi: https://doi.org/10.1101/2020.03.06.981241; this version posted September 8, 2020. The copyright holder for this preprint (which was not certified by peer review) is the author/funder, who has granted bioRxiv a license to display the preprint in perpetuity. It is made available under aCC-BY-NC-ND 4.0 International license.

Figures

A

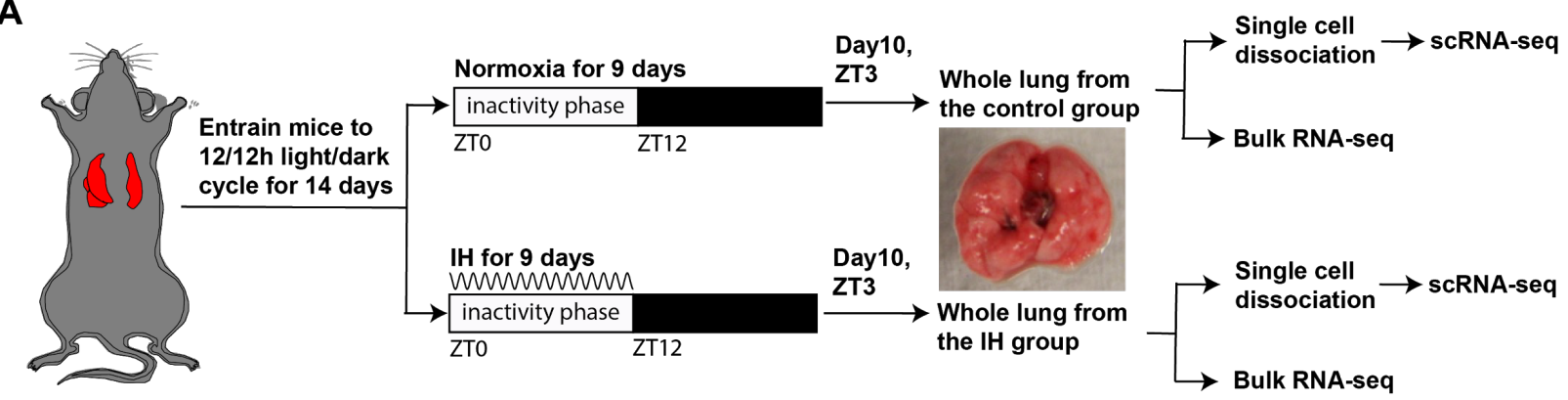

B

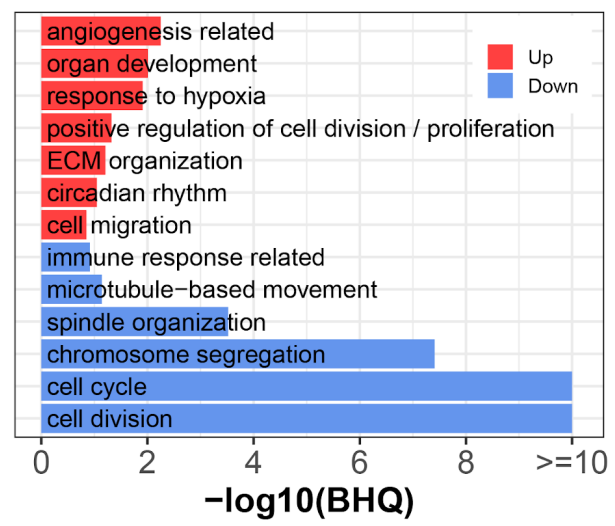

C

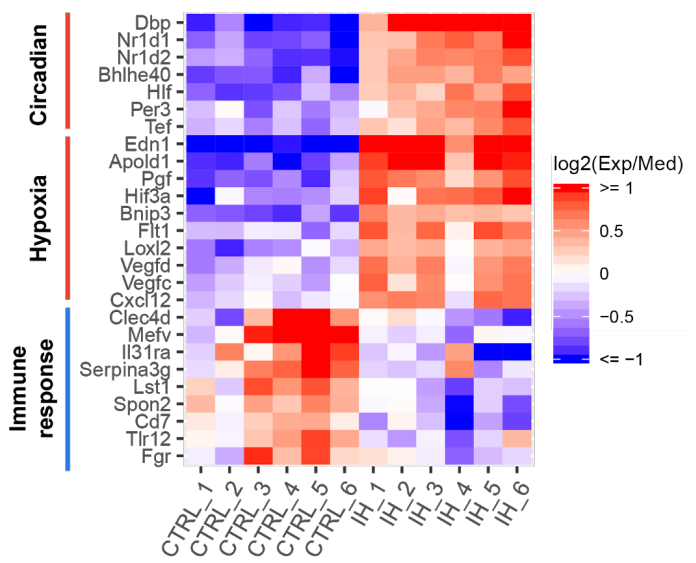

Fig. 1. Short-term exposure to intermittent hypoxia reshapes circadian and immune pathways in the lung. (A) Schematic of IH protocol. Mice are entrained to the same $12 \mathrm{~h}: 12 \mathrm{~h}$ light:dark cycle for 14 days prior to $\mathrm{IH}$ exposure. Mice are exposed to normoxia (controls) or intermittent episodes of hypoxia (21 to $6 \%$ oxygen saturation) followed by recovery to $21 \%$ oxygen over the entire $12 \mathrm{~h}$ inactivity phase for 9 days. Mice are then sacrificed at ZT 3 ( $3 \mathrm{~h}$ after lights-on) on the 10th day for tissue harvest. Bulk RNAseq and scRNA-seq were performed for each group. (B) Biological processes enriched in lung from mice exposed to IH vs controls. Enrichment analysis was performed in the DAVID database, using the top 200 up and down regulated genes identified from differential expression analyses. Redundant biological processes are merged into one category. Biological processes enriched in up and down regulated genes are indicated in red and blue bars, respectively. (C) The heatmap shows the fold change of associated genes in circadian rhythm, response to hypoxia, and immune response. The red and blue indicates up and down regulated genes in the experimental group. There are six biological replicates for each group. 
A

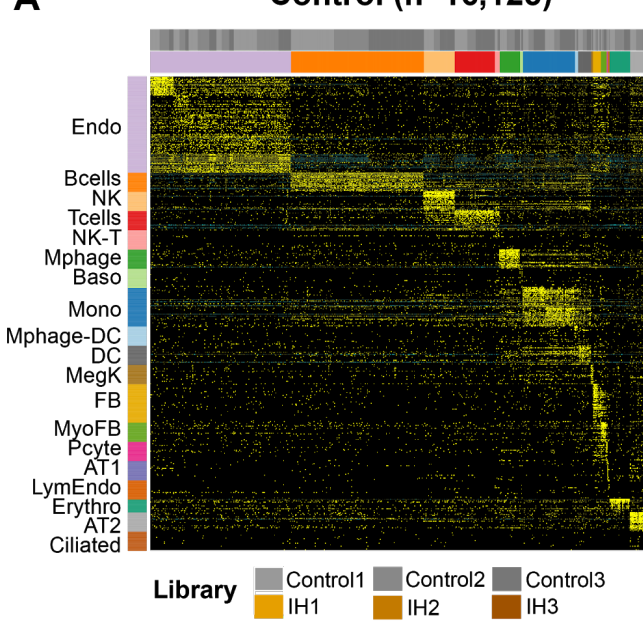

IH $(n=12,324)$

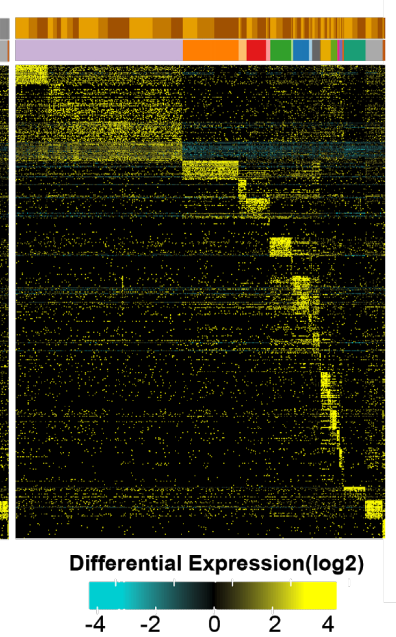

B

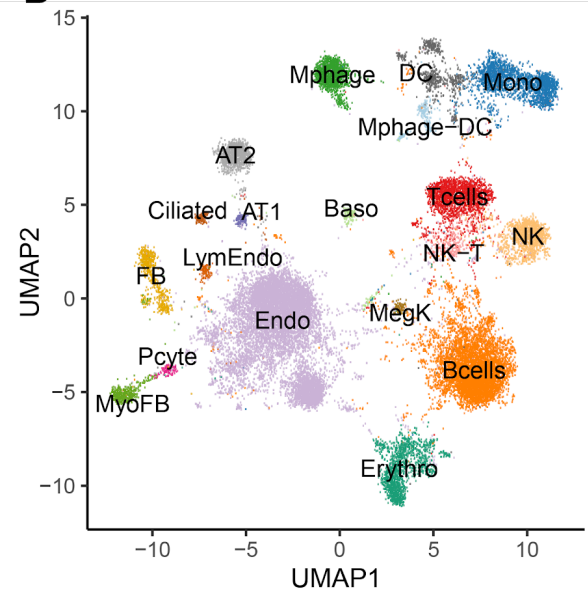

C
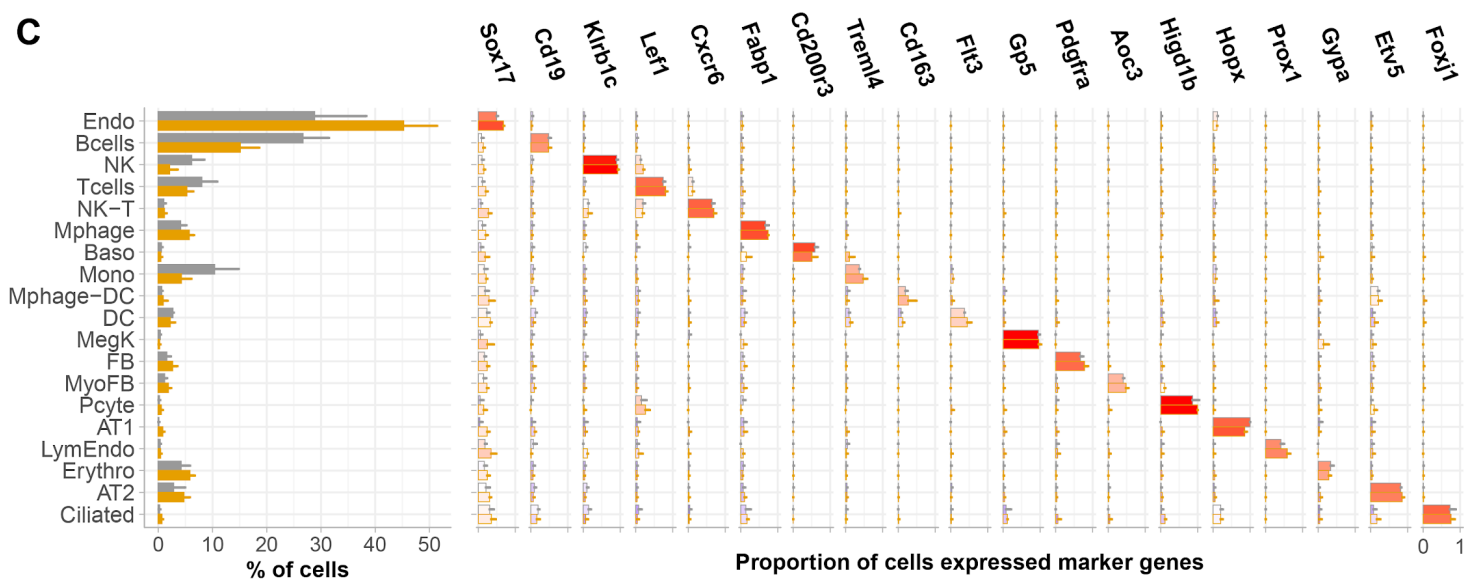

Proportion of cells expressed marker genes

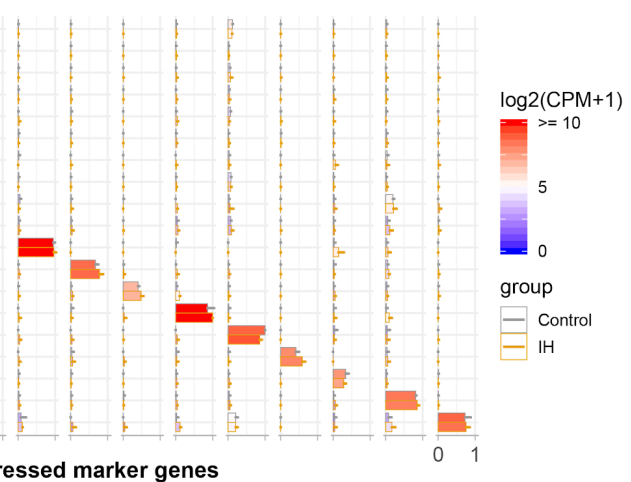

Fig. 2. Single cell sequencing identifies 19 distinct cell types in the lungs of intermittent hypoxia and control mice. (A) Heat map of AltAnalyze selected marker genes for each cell population. Columns and rows represent individual cells and marker genes, respectively. Biological replicates and cell types are indicated by the color bars on the top. cellHarmony was used to align cells from control to IH groups. (B) UMAP projection of whole lung cell populations from IH and control mice in the heatmap. (C) Percentage (left panel) and expression of marker genes (right panel) for each cell type from experimental and control mice. Error bars indicate standard deviation of cell proportion from the three replicates. The list of cell types include: endothelial cells (Endo), B cells (Bcells), natural killer cells (NK), T cells (Tcells), natural killer cells (NK), natural killer T cells (NK-T), macrophages (Mphage), basophils (Baso), monocytes (Mono), macrophages-dendritic CD163+ cells (Mphage-DC), dendritic cells (DC), megakaryocytes (MegK), fibroblasts (FB), myofibroblasts (MyoFB), pericytes (Pcyte), alveolar type I cells (AT1), lymphatic endothelial cells (LympEndo), erythroblasts (Erythro), alveolar type II cells (AT2), and ciliated cells (Ciliated). CPM indicates UMI count per million. 

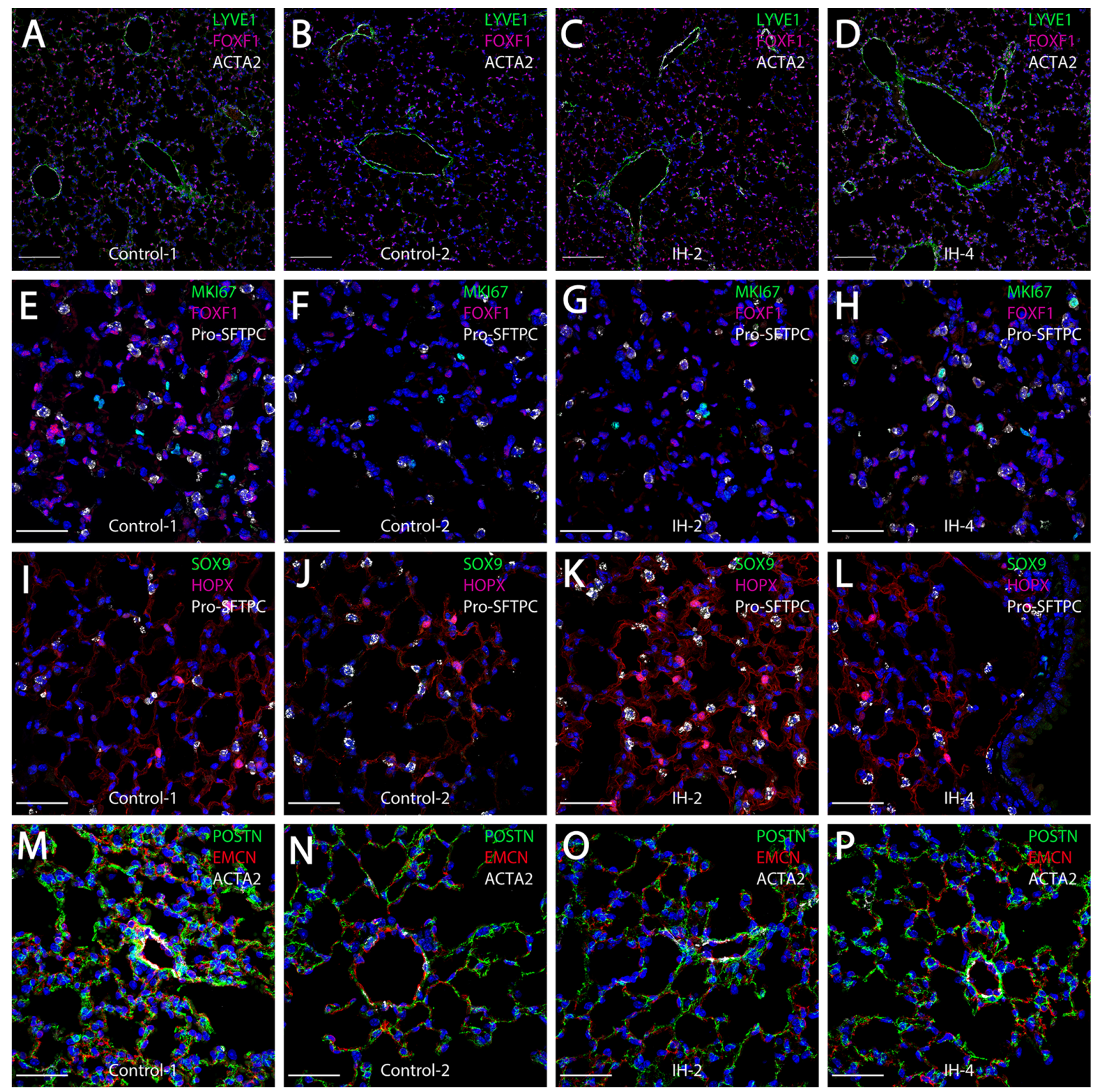

Fig. 3. Short-term exposure to intermittent hypoxia did not lead to histologic changes in the lung. (A-D) Immunostaining for LYVE1, FOXF1, and ACTA2 in mouse lung show no changes in the number or clustering of endothelial cells. (E-H) Immunostaining for MKI67, FOXF1, and Pro-SFTPC did not show changes in the number of proliferating endothelial cells. (I-L) Expression levels of SOX9, HOPX, and ProSFTPC did not demonstrate differences in the number of progenitor cells, alveolar type I cells, or alveolar type II cells. (M-P) Immunostaining for POSTN, EMCN, and ACTA2 in mouse lung did not show IHinduced changes in extracellular matrix markers. The scale bars for A-D represent $100 \mu \mathrm{m}$. The scale bars for E-P represent $40 \mu \mathrm{m}$. KEY: LYVE1 is expressed at high levels in lymphatic and vascular endothelial cells. FOXF1 and ACTA2 are expressed at high levels in vascular endothelial and smooth muscle cells, respectively. MKI67 is a marker for cell proliferation. HOPX and Pro-SFTPC show high expression levels in the alveolar type I cells and alveolar type II cells, respectively. SOX9 is a marker for

881 progenitor cells. POSTN is used to stain extracellular matrix. EMCN is a marker for capillaries and veins/venules. 
A

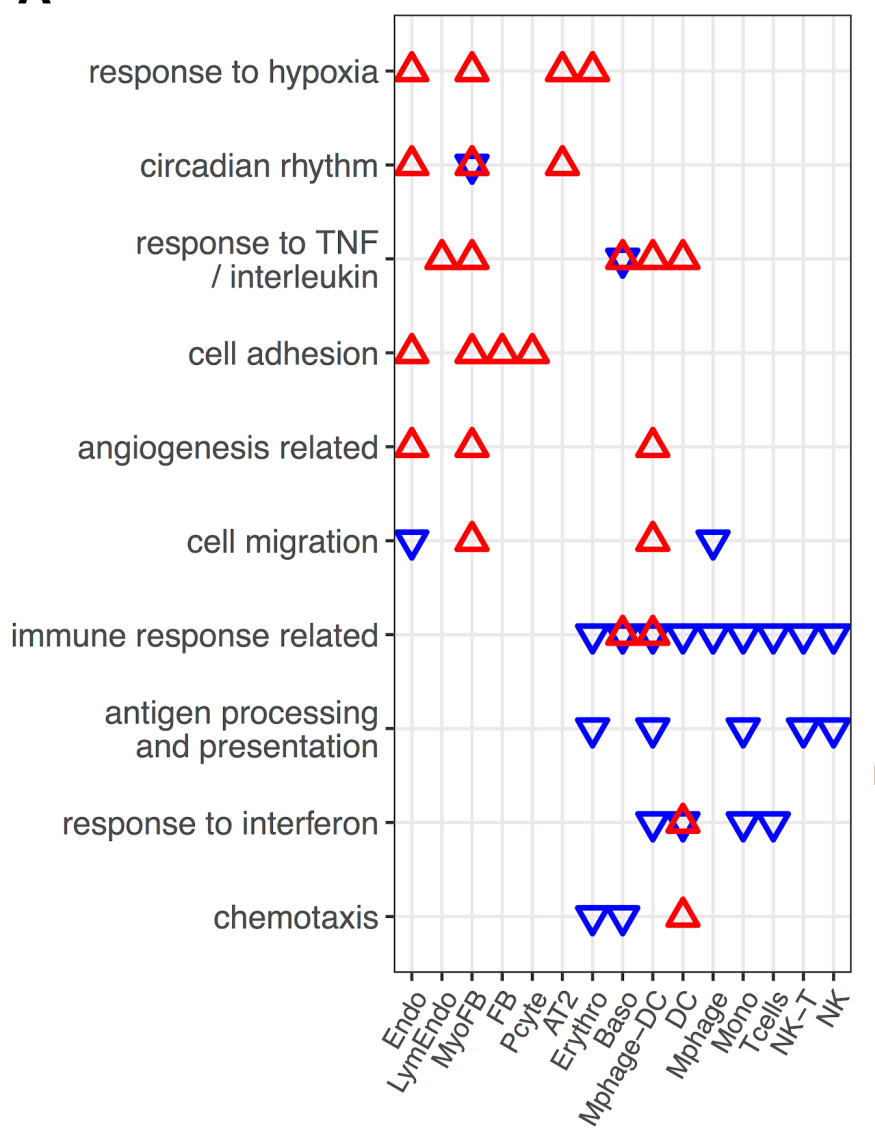

B

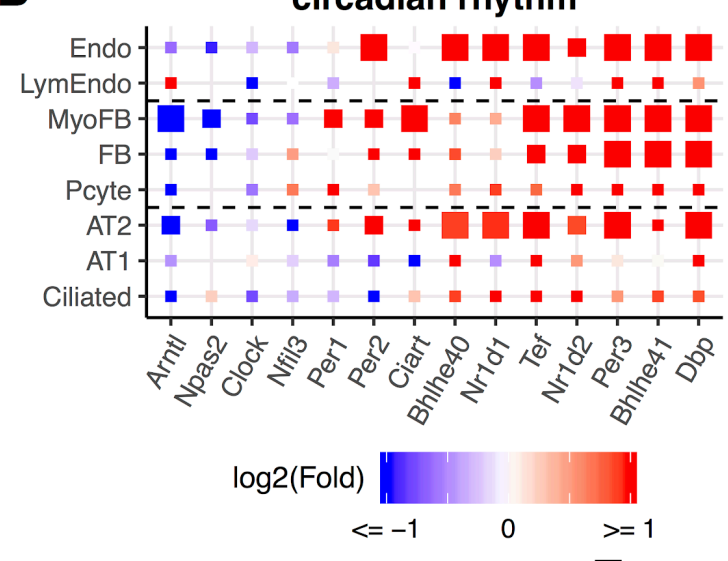

Pvalue - >= $0.05 \square[0.01,0.05) \square<0.01$

C immune response

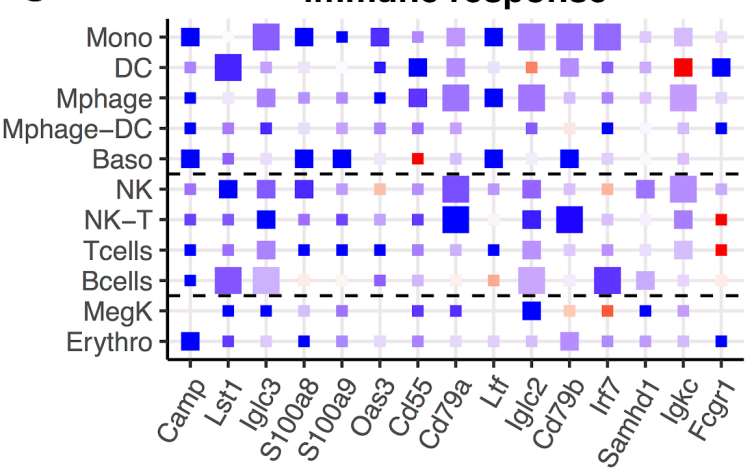

Fig. 4. Diverse expression pathways were up and down regulated in the presence of intermittent hypoxia. (A) Biological processes enriched in different cell types from lungs of mice exposed to IH vs Control. Enrichment analysis was performed in the DAVID database, using the top 200 up and down regulated genes identified from each cell type. Redundant biological processes are merged into one category. Biological processes enriched in up and down regulated genes are indicated in red and blue triangles, respectively. (B) Expression variation of well-established genes involved in circadian rhythm for endothelial, epithelial, and mesenchymal cells. (C) Expression variation of well-established genes involved in immune response for immune associated cells. For $B$ and $C$, the fold change is indicated by the color, and the P-value for differential expression is indicated by the point size. The list of cell types include: endothelial cells (Endo), B cells (Bcells), natural killer cells (NK), T cells (Tcells), natural killer cells (NK), natural killer T cells (NK-T), macrophages (Mphage), basophils (Baso), monocytes (Mono), macrophages-dendritic CD163+ cells (Mphage-DC), dendritic cells (DC), megakaryocytes (MegK), fibroblasts (FB), myofibroblasts (MyoFB), pericytes (Pcyte), alveolar type I cells (AT1), lymphatic endothelial cells (LympEndo), erythroblasts (Erythro), alveolar type II cells (AT2), and ciliated cells (Ciliated). 

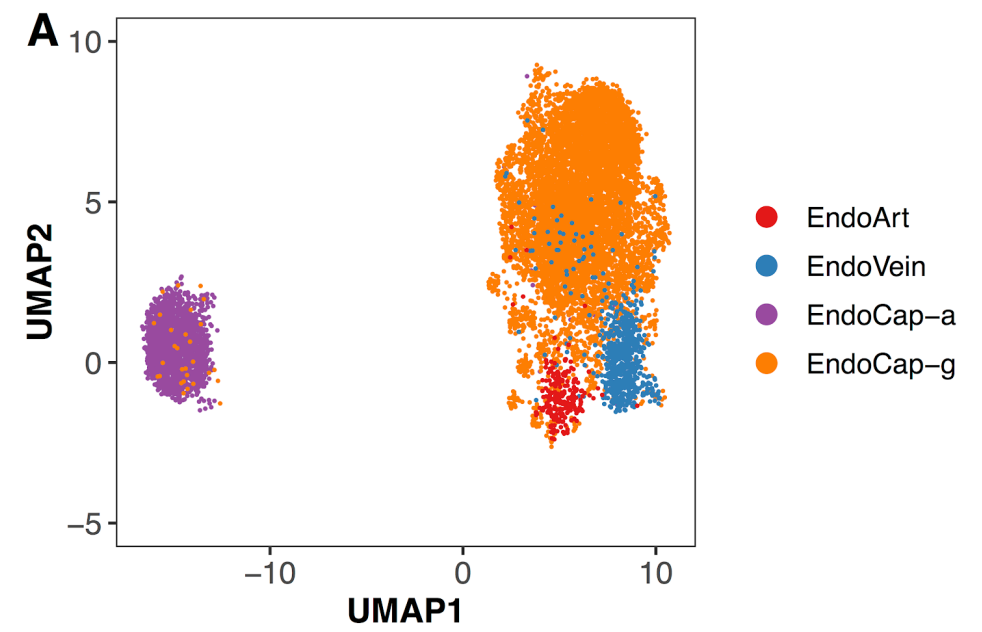

D

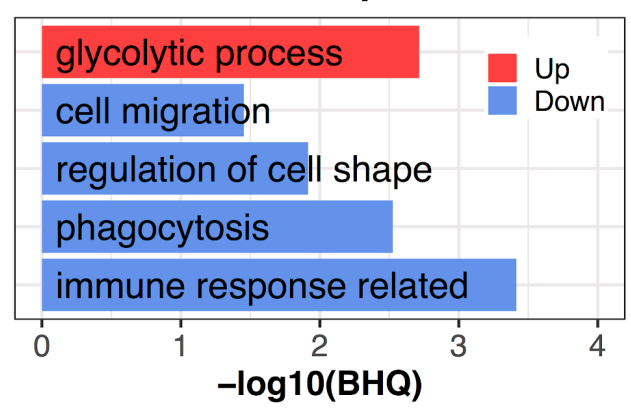

E

\section{EndoCap-g}

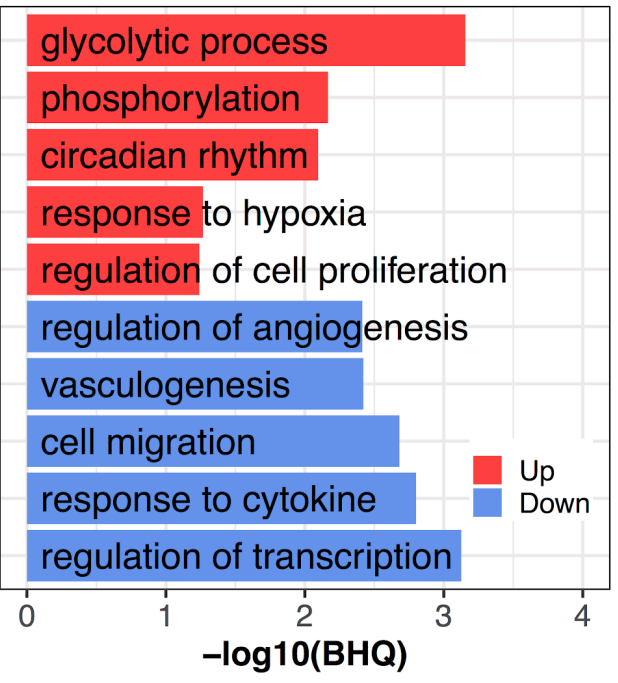

Fig. 5. Pulmonary vascular endothelial subpopulations show distinctive responses to intermittent hypoxia. (A) UMAP projection of cells from four lung endothelial subpopulations. (B) The number of differentially expressed $(B H Q<0.2)$ genes in each endothelial subpopulation from $\mathrm{IH}$ vs control. (C) Expression variation of related genes in response to $\mathrm{IH}$ is shown for each endothelial subpopulation. Biological processes enriched in capillary aerocyte (D) and capillary general $(E)$ from IH vs control mice. Enrichment analysis was performed in the DAVID database, using up and down regulated genes $(\mathrm{BHQ}<0.2)$. Redundant biological processes are merged into one category. Top 5 biological processes enriched in up and down regulated genes are indicated in red and blue bars, respectively. The list of endothelial subpopulations include endothelial artery (EndoArt), vein (EndoVein), capillary aerocytes (EndoCap-a), capillary general (EndoCap-g) cells. 
A

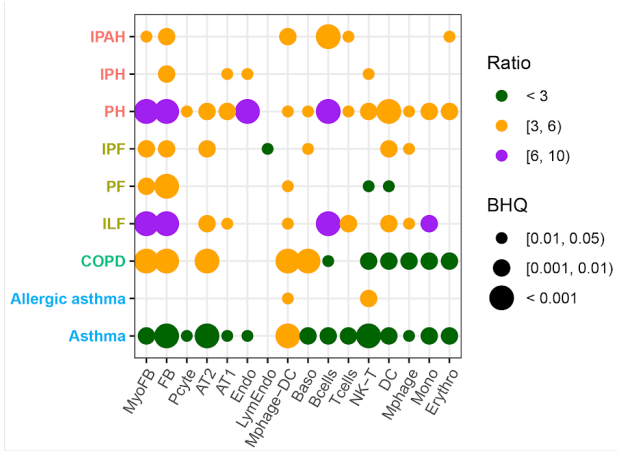

B

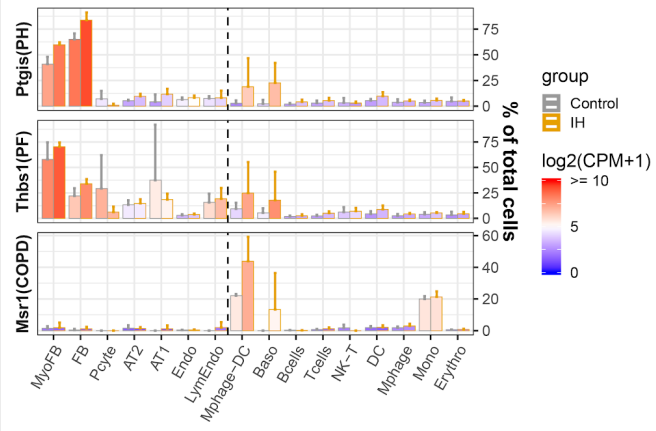

C
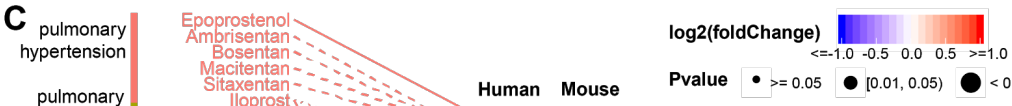

D
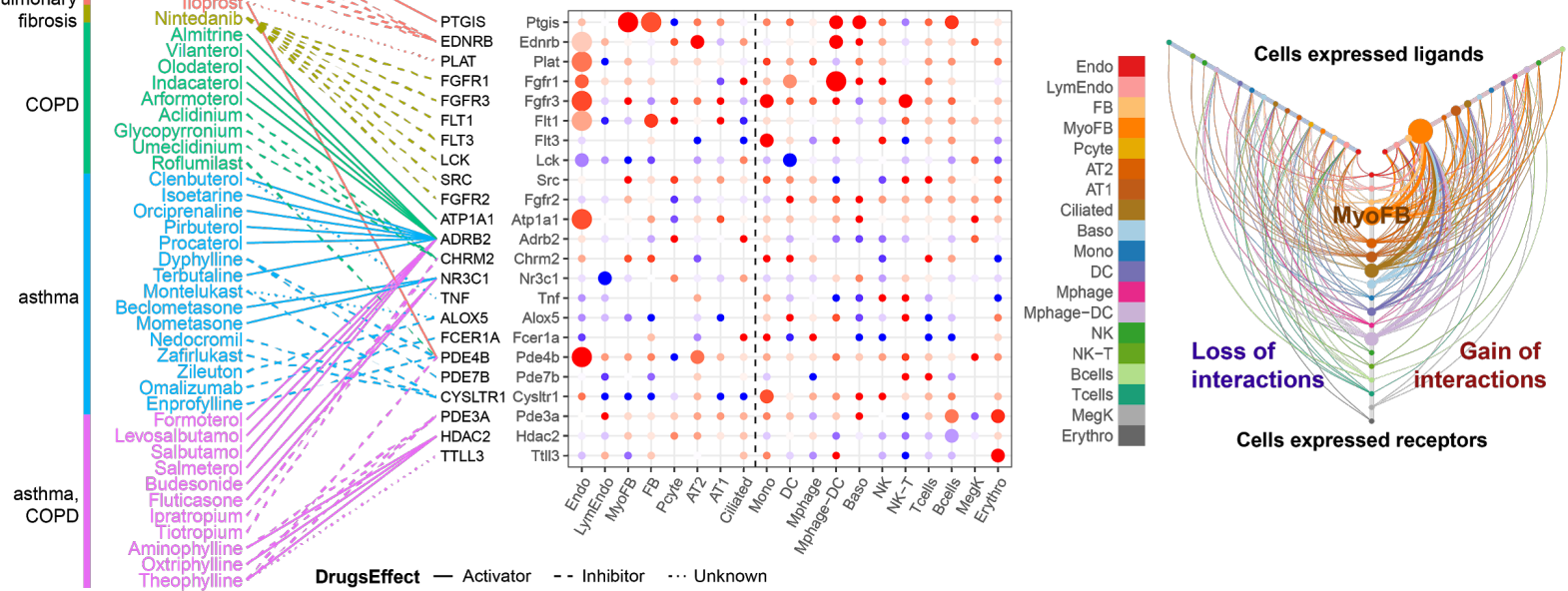

Fig. 6. Pulmonary disease-regulated genes provide clinical implications for OSA at the cellspecific level. (A) Pulmonary disease-associated genes are enriched in up regulated genes in different cell types from whole lungs of mice exposed to $\mathrm{IH}$ vs controls. Enrichment analysis was performed in DisGenet human database, using the top 200 up regulated genes identified from each cell type. The enrichment ratio is indicated by color. The point size indicates the enrichment BHQ from a fisher exact test. The pulmonary diseases include asthma, allergic asthma, chronic obstructive airway disease (COPD), interstitial lung fibrosis (ILF), pulmonary fibrosis (PF), idiopathic pulmonary fibrosis (IPF), pulmonary hypertension $(\mathrm{PH})$, idiopathic pulmonary hypertension (IPH), and idiopathic pulmonary arterial hypertension (IPAH). (B) The disease-associated genes vary in expression level and percentage of cells that express those genes. The fold change is indicated by the color, and the percentage of cells that express those genes is indicated by the height of the bar. Control and experimental groups are indicated by grey and orange box borders, respectively. (C) Dozens of pulmonary drug targets show differential expression in multiple cell types in lung from mice exposed to $\mathrm{IH}$. Drug classes used to treat different pulmonary diseases are indicated by text color. Drug effect is indicated by the line type. Fold change is indicated by the point color, and the P-value of differential expression is indicated by the point size. (D) The hive plot shows ligand-receptor interaction changes between pairs of cell types in response to $\mathrm{IH}$. Nodes indicate cell-expressed ligands (horizontal axis) or receptors (vertical axis). Size of nodes are in proportion to the number of interactions changed for the cell type. Width of lines show numbers of interactions gained (right) or lost (left) between the pairs of cell types. The list of cell types include: endothelial cells (Endo), B cells (Bcells), natural killer cells (NK), T cells (Tcells), natural killer cells (NK), natural killer T cells (NK-T), macrophages (Mphage), basophils (Baso), monocytes (Mono), macrophagesdendritic CD163+ cells (Mphage-DC), dendritic cells (DC), megakaryocytes (MegK), fibroblasts (FB), myofibroblasts (MyoFB), pericytes (Pcyte), alveolar type I cells (AT1), lymphatic endothelial cells (LympEndo), erythroblasts (Erythro), alveolar type II cells (AT2), and ciliated cells (Ciliated). 
bioRxiv preprint doi: https://doi.org/10.1101/2020.03.06.981241; this version posted September 8, 2020. The copyright holder for this preprint (which was not certified by peer review) is the author/funder, who has granted bioRxiv a license to display the preprint in perpetuity. It is made available under aCC-BY-NC-ND 4.0 International license.

\section{Supplemental figures}
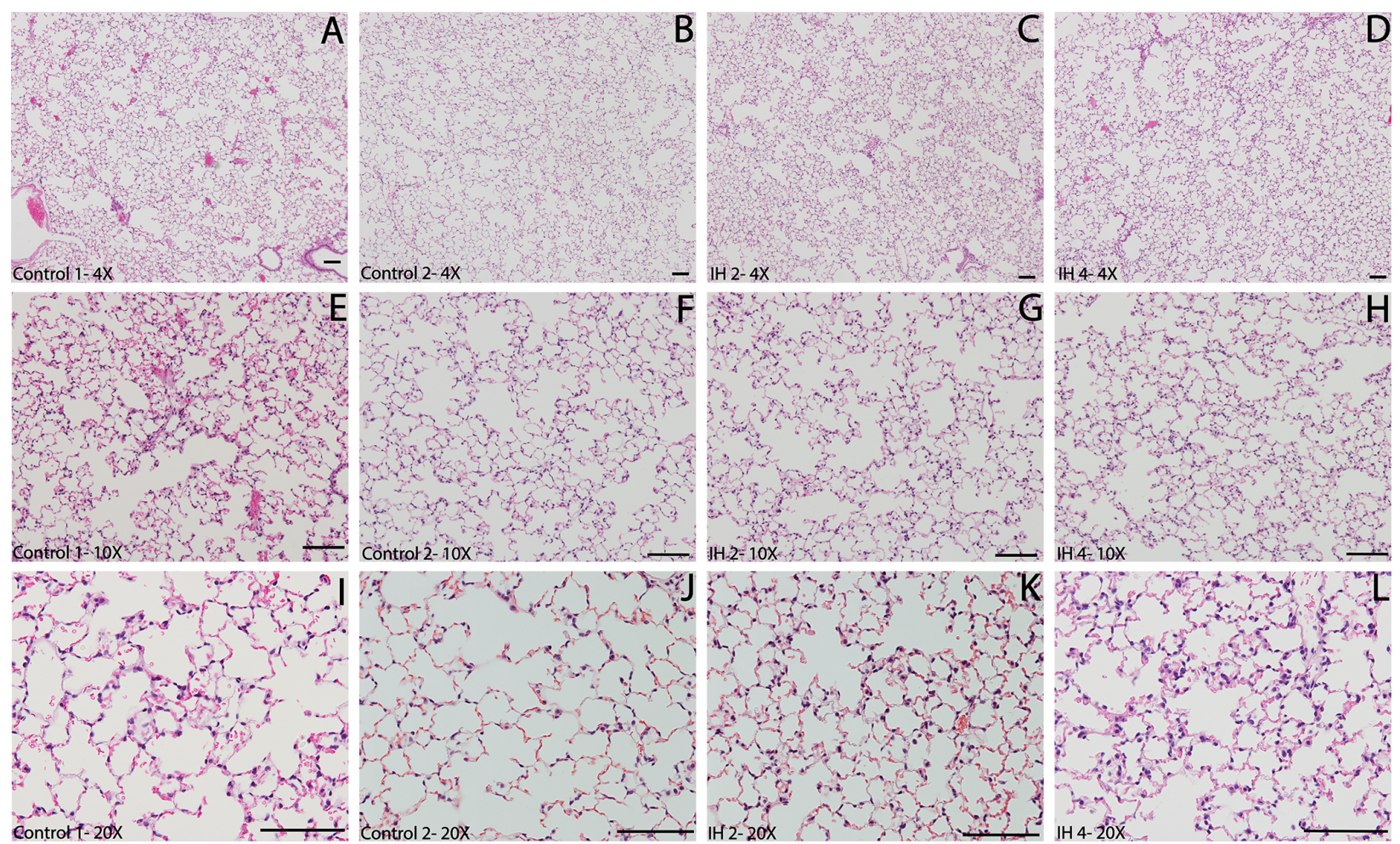

Fig. S1. Hematoxylin and eosin-stained sections of whole lung from mice exposed to IH vs stained sections were shown at $4 \times(A-D), 10 x(E-H)$ and 20x (I-L) magnification. The scale bars all correlate to $100 \mu \mathrm{m}$ width. 
bioRxiv preprint doi: https://doi.org/10.1101/2020.03.06.981241. this version posted September 8, 2020. The copyright holder for this preprint (which was not certified by peer review) is the author/funder, who has granted bioRxiv a license to display the preprint in perpetuity. It is made available under aCC-BY-NC-ND 4.0 International license.
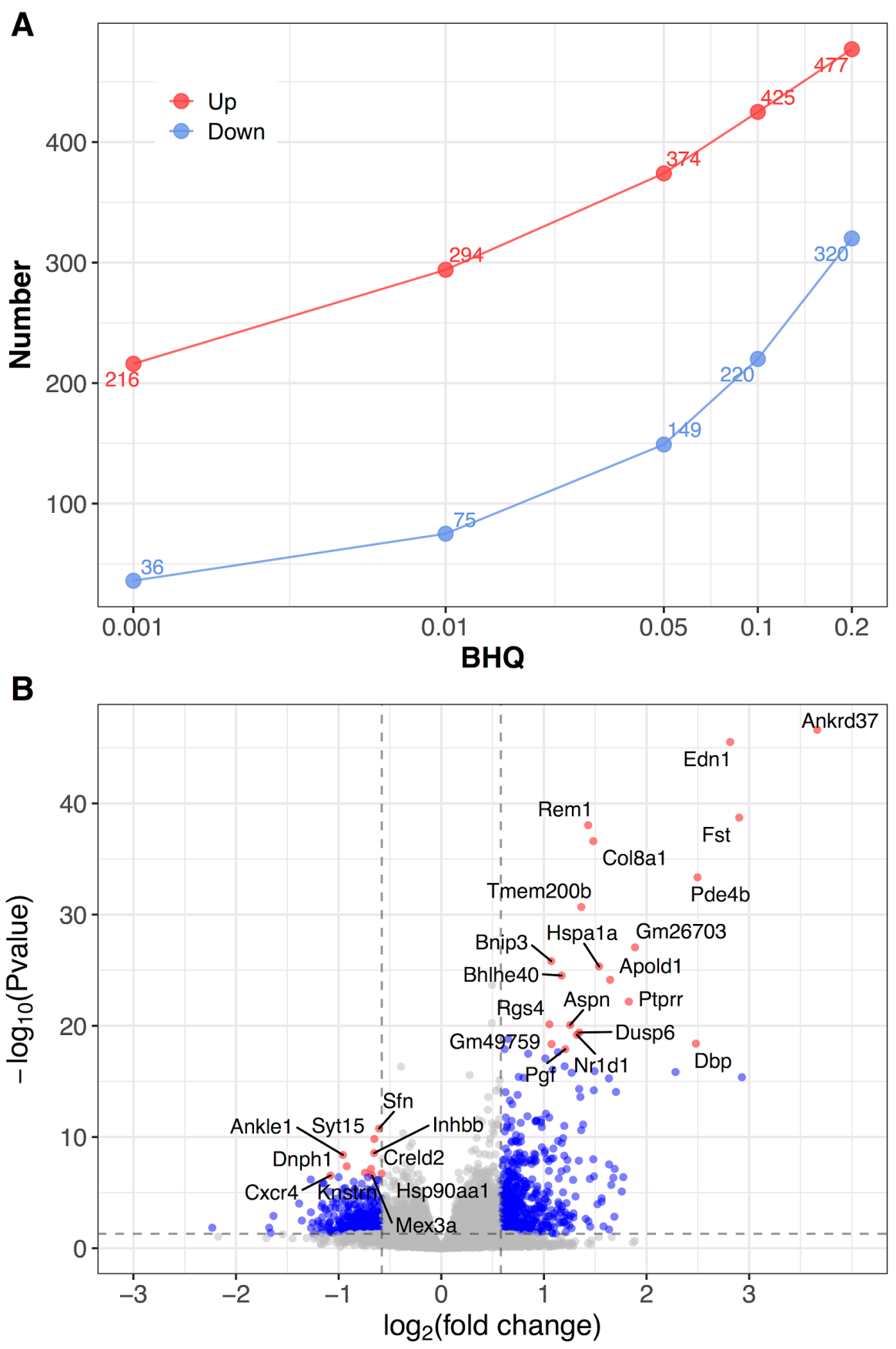

941

942

943

944

945

946
Fig. S2. Differentially expressed genes from bulk RNA-seq analysis of whole lung from mice exposed to IH vs controls. (A) At series BHQ cut-offs, the number of up and down regulated genes in response to $\mathrm{IH}$ are shown with red and blue lines, respectively. (B) A volcano plot shows the differentially expressed genes from lung of mice exposed to $\mathrm{IH}$. Ranked by P-value, top 20 up regulated genes and top 10 down regulated genes were labeled. 

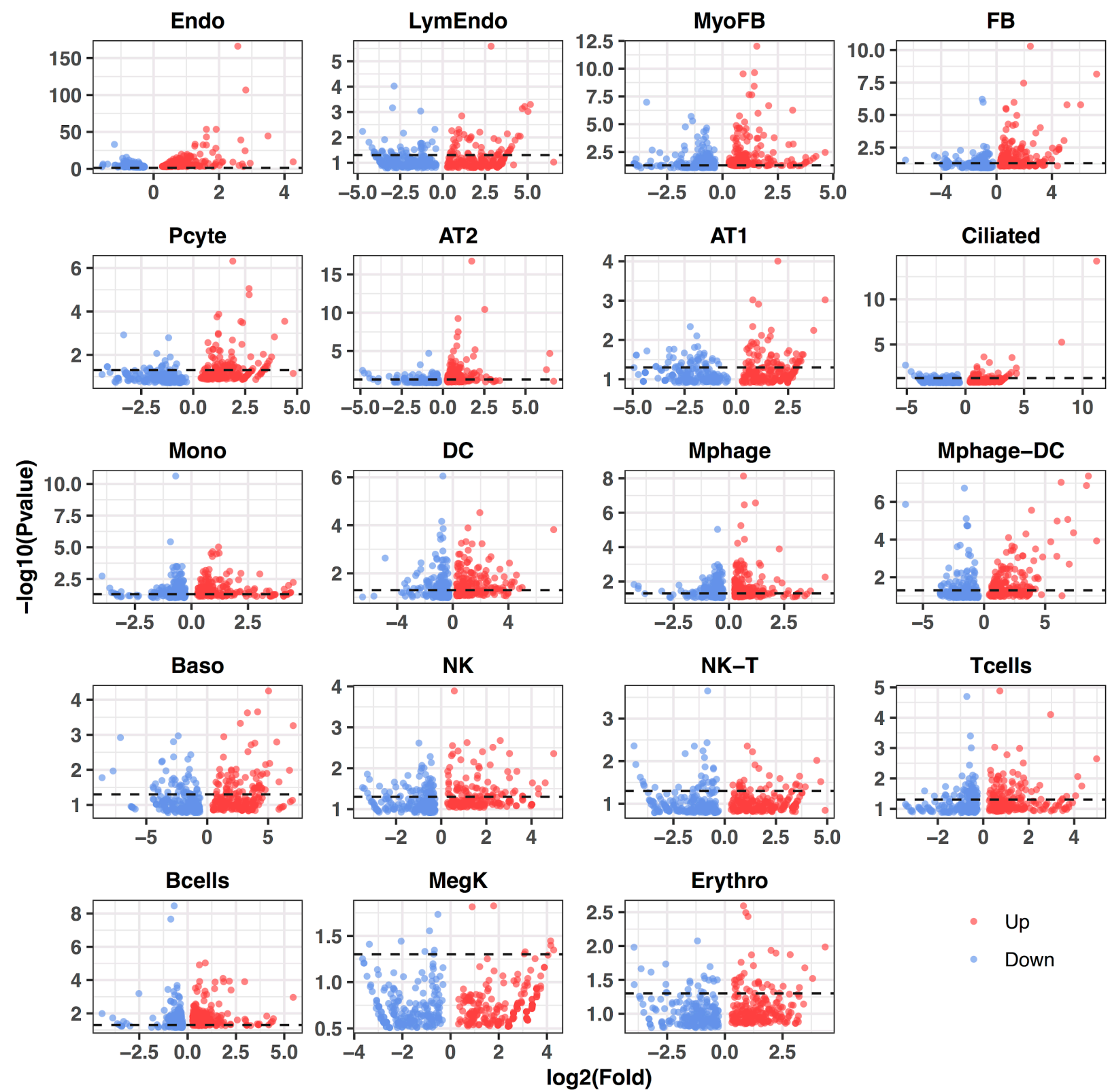

$\log 2$ (Fold) cell types. Each red or blue point indicates one up or down regulated gene. The black dashed line 
bioRxiv preprint doi: https://doi.org/10.1101/2020.03.06.981241; this version posted September 8, 2020. The copyright holder for this preprint (which was not certified by peer review) is the author/funder, who has granted bioRxiv a license to display the preprint in perpetuity. It is made available under aCC-BY-NC-ND 4.0 International license.

A

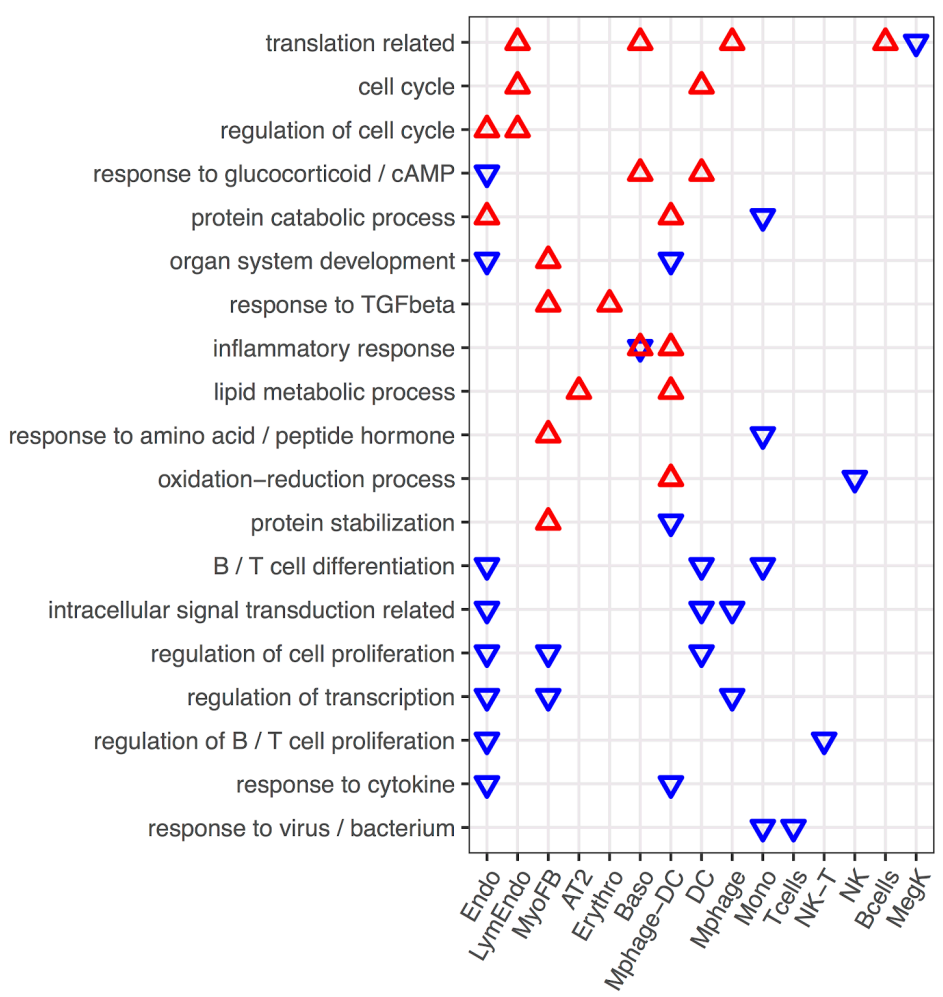

B

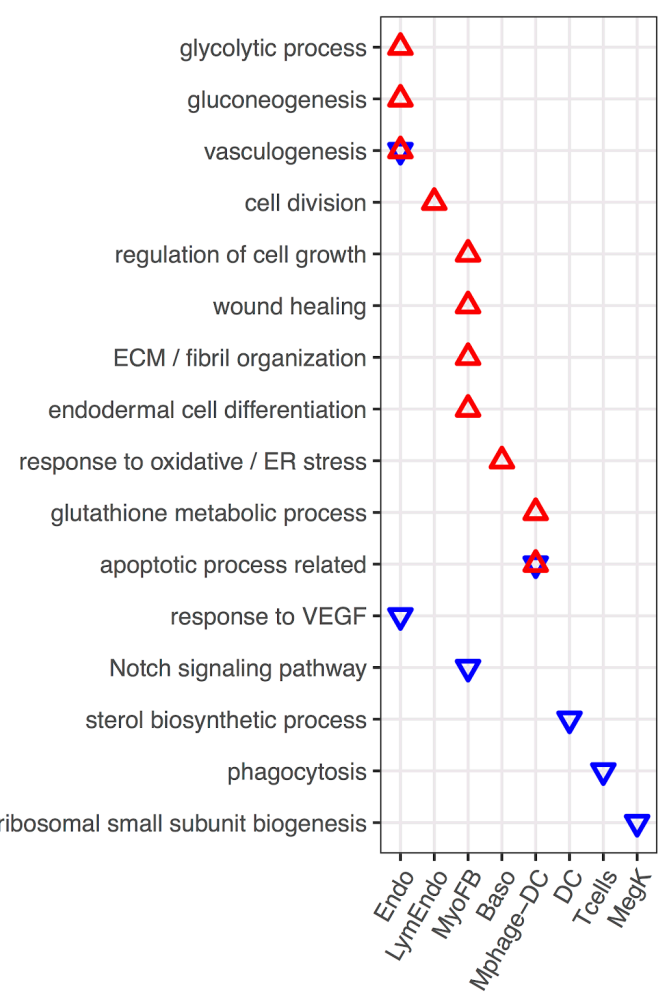

Fig. S4. Enriched biological processes from differentially expressed genes from whole mouse top 200 up and down regulated genes identified from each cell type. Redundant biological processes are merged into one category. Biological processes enriched in up and down regulated genes are indicated 

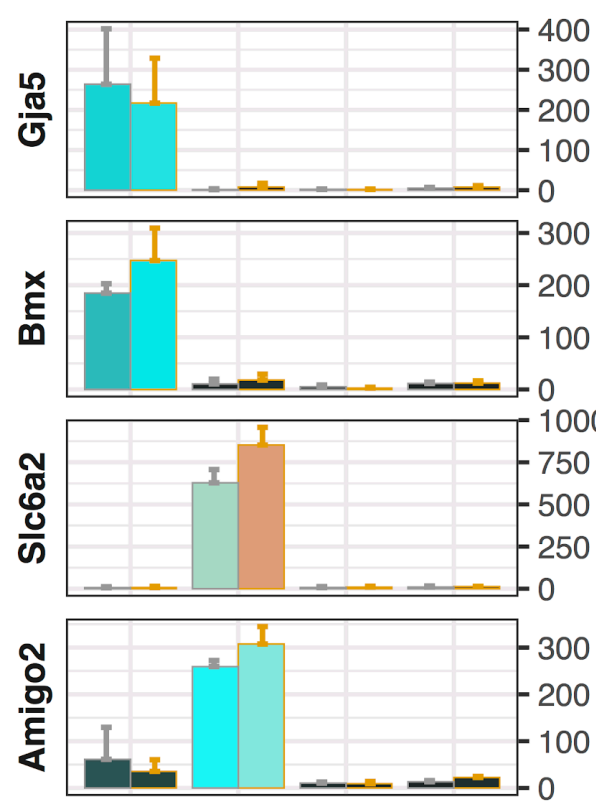

$\begin{array}{ll}\text { 을 } & \text { group } \\ \frac{7}{2} & \text { Control } \\ \mathrm{IH}\end{array}$

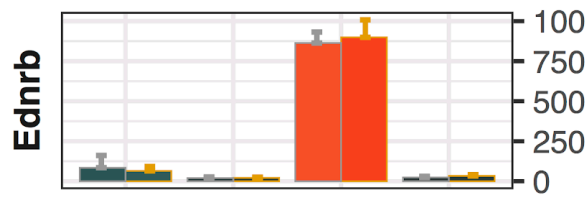

$\%$ of total cells
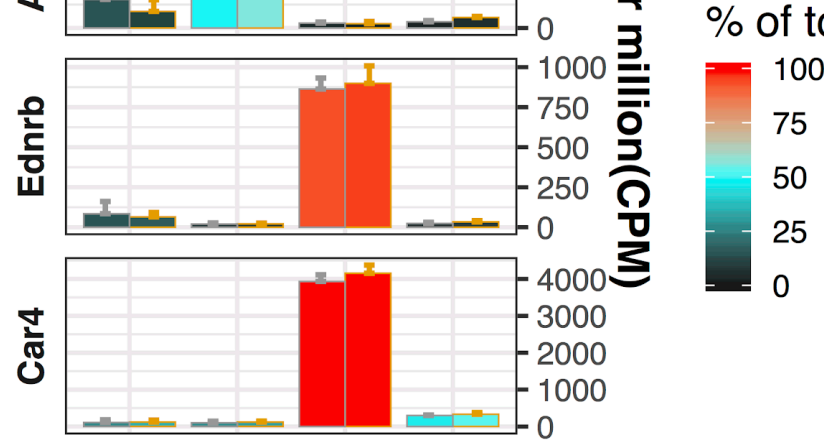

100

75

50

25
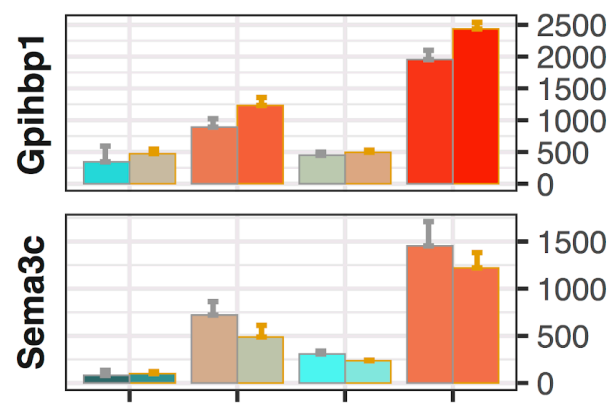

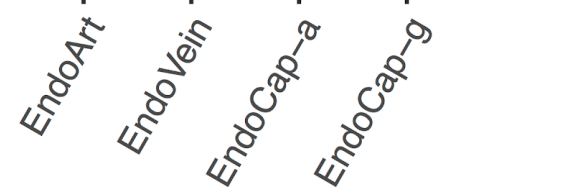

Fig. S5. Validation of endothelial subpopulation assignments with known markers. The marker genes collected from the literature and assigned endothelial subpopulations are listed on the $y$-axis and $x$ axis, respectively. The average CPM (count per million reads) of three replicates from control (grey) and $\mathrm{IH}$ (orange) mice are indicated by the height of the bar. The error bars indicate standard deviation based on the three biological replicates. The average percentage of cells with detected expression of each marker gene among the endothelial subpopulations is indicated by the heatmap color. The four (EndoCap-a), and capillary general (EndoCap-g). 
bioRxiv preprint doi: https://doi. org/10.1101/2020.03.06.981241. this version posted September 8,2020 . The copyright holder for this preprint (which was not certified by peer review) is the author/funder, who has granted bioRxiv a license to display the preprint in perpetuity. It is made available under aCC-BY-NC-ND 4.0 International license.

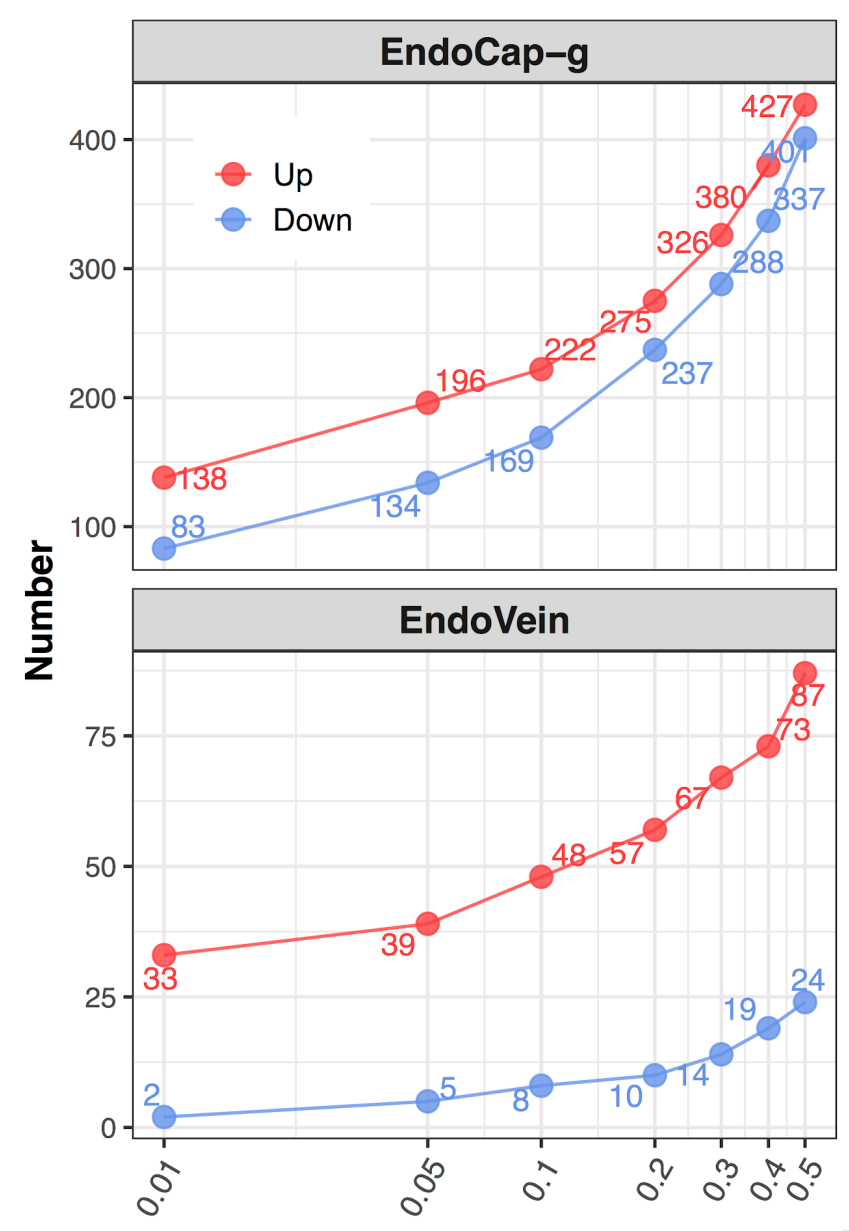

\section{BHQ}
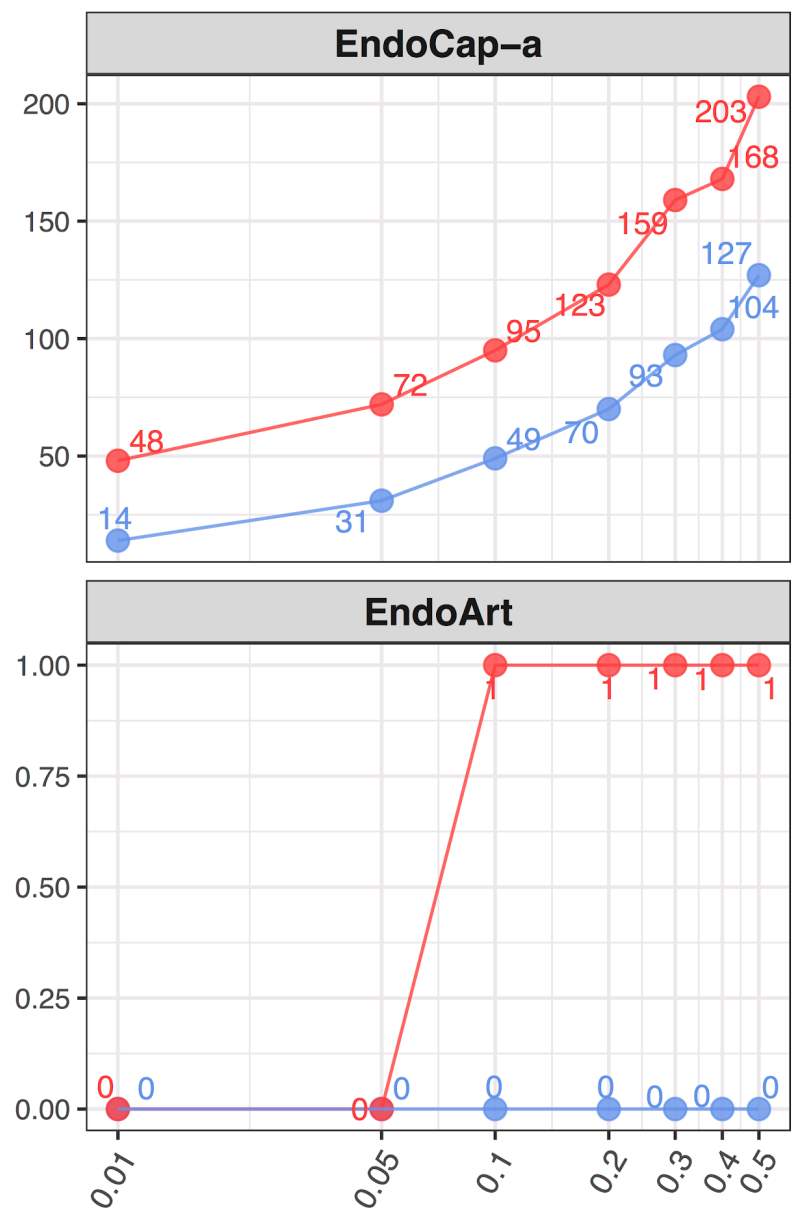

Fig. S6. Number of differentially expressed IH-responsive genes in four lung endothelial subpopulations using BHQ cut-offs. At series BHQ cut-offs, the number of up and down regulated genes in lung endothelial subpopulations as a response to $\mathrm{IH}$ are shown with red and blue lines, respectively. The four endothelial subpopulations include endothelial artery (EndoArt), vein (EndoVein), capillary aerocytes (EndoCap-a), and capillary general (EndoCap-g). 


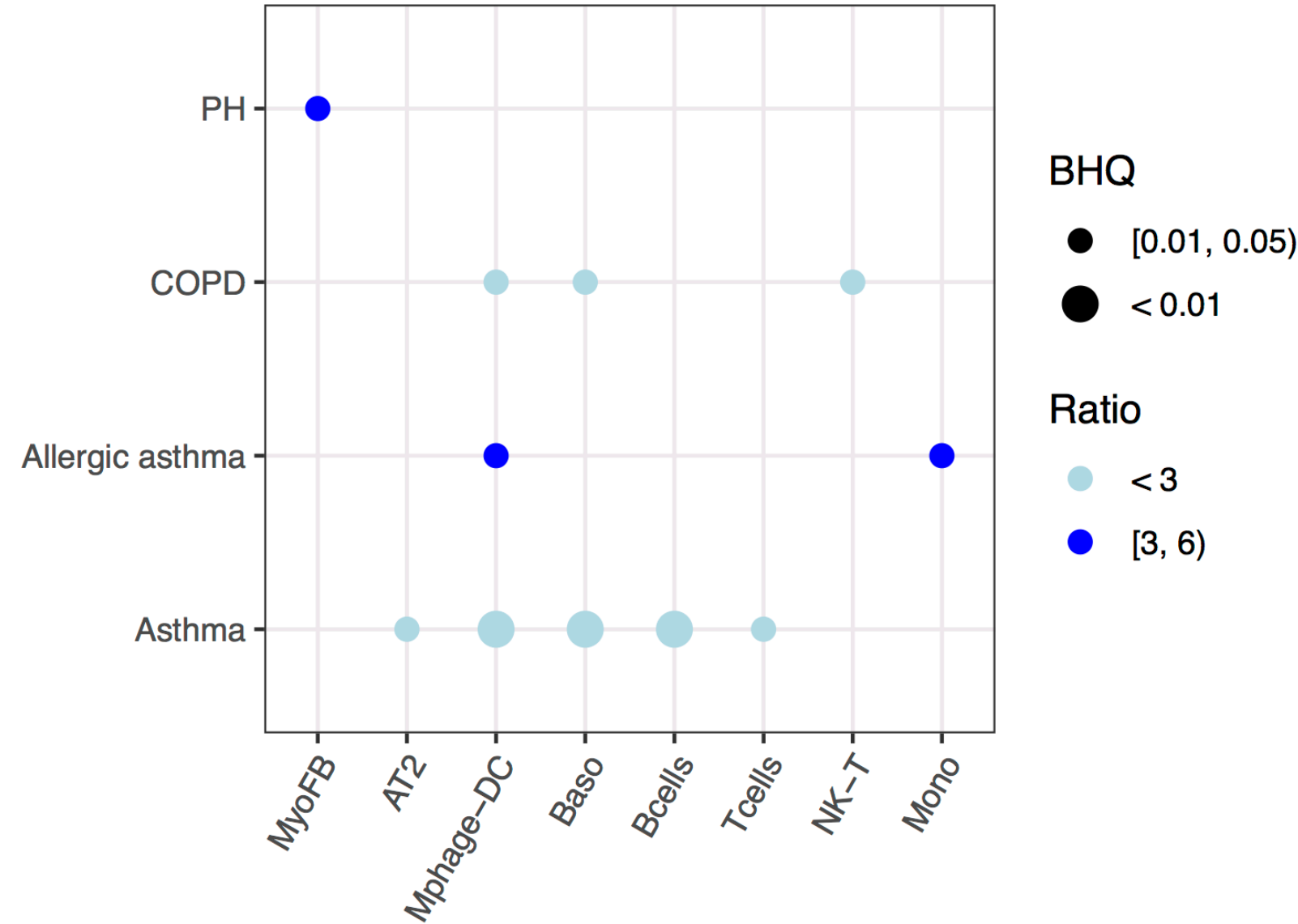

Fig. S7. Pulmonary disease-associated genes are enriched in down regulated genes in different cell types in the lung from mice exposed to IH. Enrichment analysis was performed in the DisGenet database, using the top 200 down regulated genes identified from each cell type. The average fold change of down regulated genes in each cell type for the same disease is indicated by color scale. The point size indicates the enrichment $B H Q$ value from the fisher exact test. The point color indicates the enrichment ratio. The pulmonary diseases include asthma, allergic asthma, chronic obstructive airway disease (COPD), and pulmonary hypertension. 

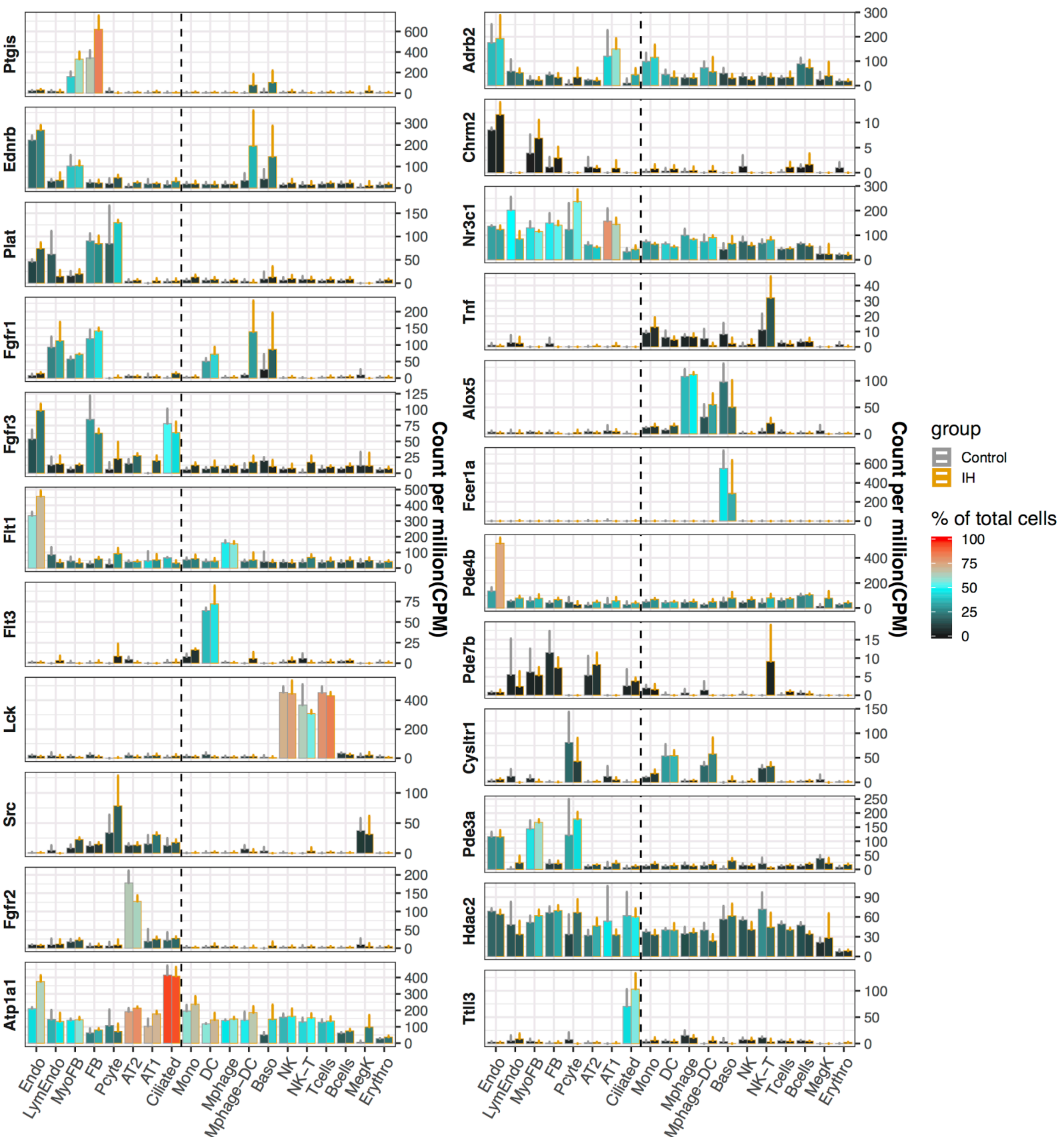

Fig. S8. Pulmonary drug targets with cell type specific expression profiles in mouse lung. The average CPM (count per million reads) of three control (grey) and $\mathrm{IH}$ (orange) replicates are indicated by the height of the bar. The error bar indicates standard deviation from the three biological replicates. The average percentage of cells with detected expression of each marker gene among specific cell types is indicated by the color scale. The list of cell types include: endothelial cells (Endo), B cells (Bcells), natural killer cells (NK), T cells (Tcells), natural killer cells (NK), natural killer T cells (NK-T), macrophages (Mphage), basophils (Baso), monocytes (Mono), macrophages-dendritic CD163+ cells (Mphage-DC), dendritic cells (DC), megakaryocytes (MegK), fibroblasts (FB), myofibroblasts (MyoFB), pericytes (Pcyte), alveolar type I cells (AT1), lymphatic endothelial cells (LympEndo), erythroblasts (Erythro), alveolar type II cells (AT2), and ciliated cells (Ciliated). 


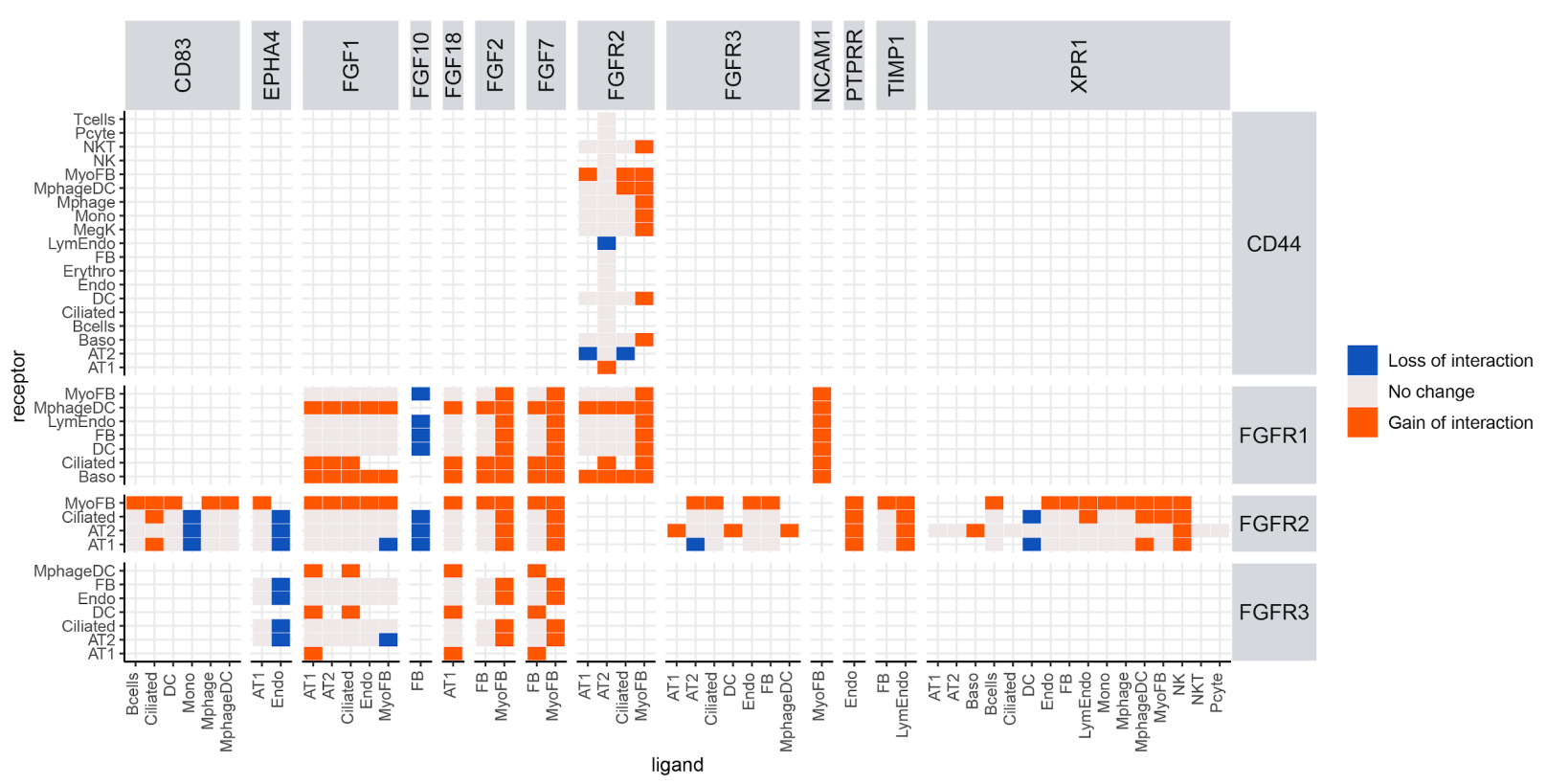

Fig. S9. Ligand-receptor interaction analysis reveals a major role for myofibroblasts in activating the FGF signaling pathway as a response to IH. Heatmap shows ligand-receptor pair interaction changes in different cell pairs. Orange indicates a new interaction found in response to $\mathrm{IH}$ but not in control samples (gain of interaction), and blue indicates an interaction found in controls but not in response to IH (loss of interaction). Myofibroblasts show a gain of interaction between FGF2, FGF7 and NCAM1 to the FGF receptor in multiple cell types, indicating its role in activating the FGF signaling pathway in response to $\mathrm{IH}$. 
Other Supplementary Material for this manuscript includes the following:

1000 Data file $\mathbf{S 1}$ contains the following supplementary tables:

1001 Table S1 (Microsoft Excel format). The alignment statistics of bulk RNA-seq data for IH

1002 exposure and control mice.

1003 Table S2 (Microsoft Excel format). Top 200 up and down regulated genes in mice lung under IH 1004 exposure from the bulk RNA-seq data.

1005 Table S3 (Microsoft Excel format). DAVID enriched biological processes and merged categories 1006 using top 200 differential expression genes from the bulk RNA-seq data.

1007 Data file S2 contains the following supplementary tables:

1008 Table S4 (Microsoft Excel format). The alignment statistics of scRNA-seq data for IH exposure 1009 and control mice.

1010 Table S5 (Microsoft Excel format). The marker genes list for 25 clusters from AltAnalyze

1011 analysis of scRNA-seq data from IH exposure mice.

1012 Table S6 (Microsoft Excel format). The annotated cell types for 25 cellHarmony aligned clusters 1013 of scRNA-seq data from $\mathrm{IH}$ exposure and control mice.

1014 Table S7 (Microsoft Excel format). Top 200 up and down regulated genes in 19 detected lung 1015 cell types under IH exposure from the scRNA-seq data.

1016 Table S8 (Microsoft Excel format). DAVID enriched biological processes and merged categories 1017 using top 200 differential expression genes in each lung cell type under IH exposure.

1018 Data file S3 contains the following supplementary tables:

1019 Table S9 (Microsoft Excel format). The marker genes list for 6 clusters from AltAnalyze analysis 1020 of scRNAseq-data from annotated vascular endothelial cells of IH exposure mice.

1021 Table S10 (Microsoft Excel format). The annotated endothelial subpopulations for 6

1022 cellHarmony aligned clusters of scRNA-seq data from annotated vascular endothelial cells of IH 1023 exposure and control mice. 
1024 Table S11 (Microsoft Excel format). Differential expression genes of 4 annotated endothelial

1025 subpopulations under IH exposure from the scRNA-seq data.

1026 Table S12 (Microsoft Excel format). DAVID enriched biological processes and merged

1027 categories using differential expression genes of lung capillary cells under IH exposure.

1028 Data file S4 contains the following supplementary tables:

1029 Table S13 (Microsoft Excel format). The pulmonary disease associated genes from top 200 up

1030 or down regulated genes in each detected lung cell-type exposed to $\mathrm{IH}$. 